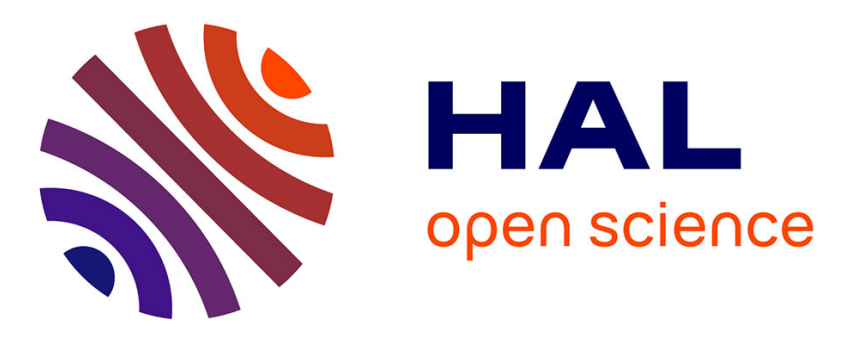

\title{
Nonlinear optical response of a gold surface in the visible range: A study by two-color sum-frequency generation spectroscopy. III. Simulations of the experimental SFG intensities
}

Bertrand Busson, L. Dalstein

\section{To cite this version:}

Bertrand Busson, L. Dalstein. Nonlinear optical response of a gold surface in the visible range: A study by two-color sum-frequency generation spectroscopy. III. Simulations of the experimental SFG intensities. Journal of Chemical Physics, 2018, 149 (15), pp.154701. 10.1063/1.5047098 . hal02111030

\section{HAL Id: hal-02111030 \\ https://hal.science/hal-02111030}

Submitted on 25 Apr 2019

HAL is a multi-disciplinary open access archive for the deposit and dissemination of scientific research documents, whether they are published or not. The documents may come from teaching and research institutions in France or abroad, or from public or private research centers.
L'archive ouverte pluridisciplinaire HAL, est destinée au dépôt et à la diffusion de documents scientifiques de niveau recherche, publiés ou non, émanant des établissements d'enseignement et de recherche français ou étrangers, des laboratoires publics ou privés. 
Nonlinear optical response of a gold surface in the visible range: A study by twocolor sum-frequency generation spectroscopy. III. Simulations of the experimental SFG intensities

B. Busson, and L. Dalstein

Citation: The Journal of Chemical Physics 149, 154701 (2018); doi: 10.1063/1.5047098

View online: https://doi.org/10.1063/1.5047098

View Table of Contents: http://aip.scitation.org/toc/jcp/149/15

Published by the American Institute of Physics 


\title{
Nonlinear optical response of a gold surface in the visible range: A study by two-color sum-frequency generation spectroscopy. III. Simulations of the experimental SFG intensities
}

\author{
B. Busson ${ }^{1}$ and L. Dalstein $1,2,3$ \\ ${ }^{1}$ Laboratoire de Chimie Physique, CNRS, Univ. Paris-Sud, Université Paris-Saclay, Bâtiment 201 P2, \\ 91405 Orsay, France \\ ${ }^{2}$ Department of Chemistry, School of Chemical Science and Engineering, KTH Royal Institute of Technology, \\ SE-100 44 Stockholm, Sweden \\ ${ }^{3}$ Institute of Physics, Academia Sinica, Taipei 11529, Taiwan
}

(Received 5 July 2018; accepted 12 September 2018; published online 15 October 2018)

\begin{abstract}
We model the amplitude line shape and absolute phase of the infrared-visible sum-frequency signals produced by a thiolated polycrystalline gold surface as a function of the visible wavelength. We follow two hypotheses: in the interband scenario, the resonant features are attributed to interband transitions, whereas in the effective surface state scenario, they stem mostly from the excitation of surface transitions. We find that both scenarios lead to a satisfactory account of the experimental data and that only free electrons may spill out of the gold bulk, as expected. For the interband scenario, the balance between free and bound electron contributions to sum-frequency generation has to be adjusted to fit the data. The surface transitions are shown to take their origin inside gold and we investigate the surface states involved in such transitions, with a comparison to the silver surfaces. We finally provide a work program dedicated to discriminate between the two scenarios. Published by AIP Publishing. https://doi.org/10.1063/1.5047098
\end{abstract}

\section{INTRODUCTION}

In Paper I of this series, ${ }^{1}$ we have measured the experimental effective nonlinear susceptibility of a polycrystalline gold film in an infrared-visible Sum-Frequency Generation (SFG) experiment while tuning the visible wavelength over the visible range (435-705 nm) (two-color Sum-Frequency Generation, $2 \mathrm{C}$-SFG). Using the vibrationally resonant response of an adsorbed thiol monolayer, we could extract the absolute phase and amplitude line shape of gold in these conditions. In Paper II, ${ }^{2}$ we have developed a model for the simulation of the nonlinear SFG response of a gold surface (or a metal surface in general) in such experiments, taking into account both free and bound electrons together. In this paper, we propose to build on this theory to account for the experimental data.

Simulations of the silver and gold 2C-SFG response show that they theoretically should have comparable amplitudes. ${ }^{2}$ This is not the case experimentally ${ }^{3}$ as the signals from gold appear much more intense than those for silver, for a visible wavelength of $532 \mathrm{~nm}$, for example. The silver response is well modelled by a free electron behavior and will serve as a reference case for gold in the following. In order to explain the experimental difference in magnitude between both metals, we have to take into account an additional phenomenon specific to gold. We therefore separate the gold response into a regular contribution, calculated in the same way as for silver, and a corrective term, which accounts for the specificities of gold but is absent for silver. We may follow two tracks: according to the first one, hereafter called the interband scenario, we consider that the difference solely originates in the presence of gold interband transitions in the middle of the visible range. In other words, the nonlinear response of gold in terms of surface and bulk contributions is the only source of SFG photons in the system. We evaluate the nonlinear response to the $2 \mathrm{C}-\mathrm{SFG}$ experiment in the theoretical frame of Refs. 4 and 5, where the metal is described by its free and bound electron densities and polarizabilities. We investigate three schemes for the nonlinear optical response, which differ by their electron density profiles as a function of depth and their associated coupling between free and bound contributions. Second, we explore the possibility that the excitation of surface states is involved in the surface enhancement of the gold response. ${ }^{6}$ As the properties of these states may vary according to their nature ${ }^{7}$ and to the local crystalline order and orientation of the gold surface, ${ }^{8}$ we consider a general description of such surface transitions with Lorentzian profiles.

In this paper, we therefore simulate the experimental data under these two assumptions. We show that both descriptions may account for the experimental results. The surface state hypothesis gives more accurate simulations but involves several parameters adjusted by curve fitting. The interband scenario only involves two adjustable parameters and also provides a good approximation of the data after adjustment of the balance between free and bound electron terms. After discussing the properties of the surface states involved in the surface transitions for gold and silver, we finally propose a work program in order to discriminate between both scenarios through dedicated SFG experiments. 


\section{REVIEW OF THE MODEL FOR THE NONLINEAR RESPONSE OF GOLD}

Two light beams, modeled by plane waves at frequencies $\omega_{1}$ (visible, angle of incidence $\theta_{1}$ ) and $\omega_{2}$ (infrared, $\theta_{2}$ ) propagate in air (medium $1, \varepsilon^{\text {air }}=1$ ) and coincide at the surface of gold (medium 2, dielectric function $\varepsilon^{\text {gold }} \equiv \varepsilon$, with $\varepsilon_{\mathrm{i}}=\varepsilon\left(\omega_{\mathrm{i}}\right)=\left(\mathrm{n}_{\mathrm{i}}\right)^{2}$, where $\mathrm{n}$ is the complex index of refraction of gold), supposed isotropic. The SFG beam $\left(\omega_{3}=\omega_{1}+\omega_{2}\right)$ is detected in reflection at the phase matching angle $\theta_{3}: \omega_{3} \sin \theta_{3}=\omega_{1} \sin \theta_{1}+\omega_{2} \sin \theta_{2}$. All beams are p-polarized.

We briefly recall, here and in Appendix A, the previous results on the simulation of the nonlinear response of gold. ${ }^{2}$ The SFG intensity in the far field is given by

$$
\mathrm{I}_{\mathrm{p}}\left(\omega_{3}\right)=\frac{8 \pi^{3}\left(\omega_{3}\right)^{2}}{\mathrm{c}^{3} \cos ^{2} \theta_{3}}\left|\chi_{\mathrm{I}, \mathrm{ppp}}^{\text {eff }}\right|^{2} \mathrm{I}_{\mathrm{p}}\left(\omega_{1}\right) \mathrm{I}_{\mathrm{p}}\left(\omega_{2}\right)
$$

with

$$
\mathrm{I}_{\mathrm{p}}\left(\omega_{\mathrm{i}}\right)=\frac{\mathrm{c}}{2 \pi}\left|\mathrm{E}_{\mathrm{p}}\left(\omega_{\mathrm{i}}\right)\right|^{2}
$$

The effective nonlinear susceptibility $\chi^{\text {eff }}$ [ [superscripts (2) are dropped in the following] accounts for the response of gold at the interface, the response of the monolayer having been used as an internal reference in order to calibrate that of gold. We have seen ${ }^{2}$ that it encompasses several terms originating from the bulk ( $\chi_{\text {bulk }}^{\text {eff }}$ ) and the surface ( $\chi^{\text {eff }}$ s), decomposed in parallel and perpendicular contributions to the surface. Up to now, we have considered that the surface term is defined solely as the contribution of the bulk polarization confined in the interface region and made essentially different from the pure bulk contribution by the rapid variations of the material properties between medium 1 and medium 2. However, it is also possible to consider a pure surface contribution, i.e., not related to a bulk process. ${ }^{9}$ In the isotropic case, the surface symmetries alone do not form new nonvanishing dipolar terms in the nonlinear susceptibility. Such an additional term would suppose that there exists a surface process (i.e., not belonging to the bulk) to generate it. The most obvious case would be the involvement of surface states. ${ }^{7}$ In this scenario, specific surface transitions between occupied and empty states would give rise to a resonant additional source of SFG radiation. Several publications have attributed the resonances they observed by second harmonic generation (SHG) on a metal surface to a surface state. ${ }^{10-12}$ Such a state could also stem from the adsorption process of the thiol, i.e., from the Au-S link, similar to the Pt-CO system ${ }^{13}$ (so-called interface or extrinsic surface states, as recalled in the discussion below). Resonance with a surface state will enable an additional nonvanishing term to the surface nonlinear susceptibility, of dipolar nature $\left(\chi_{D}{ }^{\text {eff }}\right)$,

$$
\chi_{\mathrm{i}}^{\mathrm{eff}}=\chi_{\mathrm{S}}^{\mathrm{eff}}+\chi_{\mathrm{bulk}}^{\mathrm{eff}}+\chi_{\mathrm{D}}^{\mathrm{eff}} \equiv \chi_{\mathrm{gold}}^{\mathrm{eff}}+\chi_{\mathrm{D}}^{\mathrm{eff}} .
$$

The effective nonlinear susceptibilities $\chi^{\text {eff }}$ relate to the local nonlinear susceptibilities $\chi$ through the Fresnel coefficients, which account for the transition between the far field and local field.

Gold is first described in the following as the superposition of two electron populations, a free electron density $\left(\mathrm{n}_{0}{ }^{\mathrm{f}}\right)$ and a bound electron density $\left(\mathrm{n}_{0}{ }^{\mathrm{b}}\right)$, with $\mathrm{n}_{0}{ }^{\mathrm{f}}+\mathrm{n}_{0}{ }^{\mathrm{b}}=\mathrm{n}_{0}$. The interband contribution from the bound electrons shall not be neglected when considering that, for a threshold around $2 \mathrm{eV},{ }^{14}$ the SFG beam in our experiments is always able to excite them. The bulk dielectric function of gold is therefore written as ${ }^{5}$

$$
\varepsilon_{\mathrm{i}} \equiv \varepsilon\left(\omega_{\mathrm{i}}\right)=1+4 \pi \alpha_{i}^{\mathrm{f}} \mathrm{n}_{0}^{\mathrm{f}}+4 \pi \alpha_{\mathrm{i}}^{\mathrm{b}} \mathrm{n}_{0}^{\mathrm{b}},
$$

where $\alpha_{i}{ }^{f}$ (respectively, $\alpha_{i}^{b}$ ) represent the electronic polarizabilities of the free (respectively, bound) electrons at frequency $\omega_{\mathrm{i}}$, and separated into the two components by $\varepsilon_{i}-1=\left(\varepsilon_{i}^{f}-1\right)+\left(\varepsilon_{i}^{b}-1\right)$. The dielectric function of gold is tabulated from the work of Olmon et al. ${ }^{15}$ In order to separate the free and bound electron parts, we fit the free electron behavior by a Drude model in the near infrared range and find parameters $\omega_{\mathrm{P}}=8.80 \mathrm{eV}$ and $\Gamma=49.4 \mathrm{meV}$. In the visible range, the bound electron dielectric function follows: $\varepsilon_{\mathrm{i}}^{\mathrm{b}}=1+\varepsilon_{\mathrm{i}}-\varepsilon_{\mathrm{i}}^{\mathrm{f}}$ for $\mathrm{i}=(1,3)$. For the infrared beam, the remaining bound electron contribution $\varepsilon_{2}{ }^{\mathrm{b}}$ is supposed real $^{5}$ but unknown, we therefore perform our simulations for several values between 1.5 and 8 in order to estimate the influence of this parameter.
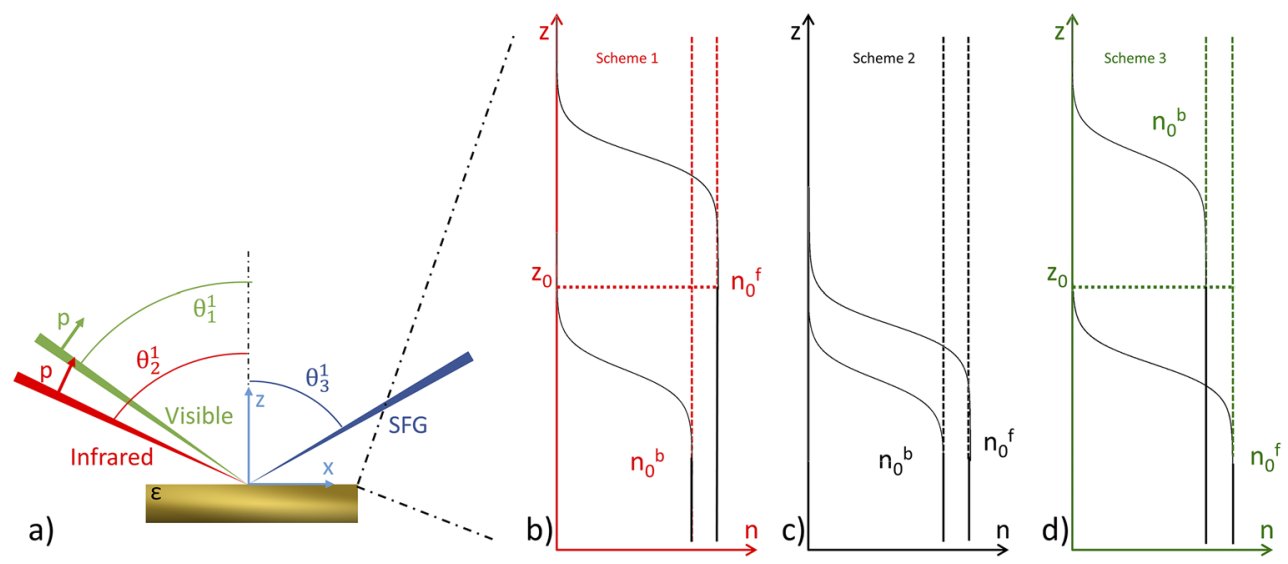

FIG. 1. (a) Sketch of the experimental configuration. [(b)-(d)] Schemes of the distributions of the electron densities $\mathrm{n}^{\mathrm{f}}(\mathrm{z})\left[\right.$ respectively, $\left.\mathrm{n}^{\mathrm{b}}(\mathrm{z})\right]$ of free $($ respectively, bound) electrons as a function of depth inside gold for schemes 1 (b), 2 (c), and 3 (d). 
The polarizabilities quantify the amplitude of the electronic response to the excitation by light, and the second order nonlinear response is therefore proportional to a product of two electronic polarizabilities. As the free and bound electronic polarizabilities differ, their contributions to the SFG signal must be evaluated separately, then summed up, immersed in a medium which the dielectric function encompasses free and bound parts together. We model the bound part by a continuous dipolium model as in Ref. 5.

The properties of the bulk term have been described before. $^{2}$ The gold surface term $\chi_{\mathrm{S}}$ is expressed in terms of Rudnick-Stern coefficients (a for perpendicular and $b$ for parallel to the surface); it depends on the dielectric functions at the three involved wavelengths and on the bulk electron densities $\mathrm{n}_{0}{ }^{\mathrm{f}}$ and $\mathrm{n}_{0}{ }^{\mathrm{b}}$. As for the perpendicular surface component, its amplitude, phase, and spectral evolution in the visible range also depend on the relative profiles of the free and bound electron densities across the interface. We have shown that they may be explicitly calculated without precise knowledge of the actual profiles in the three schemes shown in Fig. 1. The formulas are provided in Appendix A.

\section{THE INTERBAND SCENARIO}

In order to simulate the nonlinear response of gold, we first consider the case where no surface state is present (i.e., $\chi_{D}{ }^{\text {eff }}=0$ ). According to Eq. (A7), the key parameter of the model is the balance between free and bound electron contributions-in other words, the relative weights between the two electron densities $\mathrm{n}_{0}{ }^{\mathrm{f}}$ and $\mathrm{n}_{0}{ }^{\mathrm{b} 5}$. An absolute value for parameter $\mathrm{n}_{0}{ }^{\mathrm{f}}$ may be known from the atomic density of gold. The bound electron contribution is considered here as a whole. However, the valence electrons fall into two families: two electrons in the band closest to the Fermi level (upper d-band) experience interband transitions in the visible and near-UV (close to the $\mathrm{X}$ and $\mathrm{L}$ points of the Brillouin zone, respectively ${ }^{14}$ ), while the other eight electrons lie in bands with lower energies $^{16}$ (lower d-bands). A refined model explicitly separating both contributions is currently under investigation, but the impact on simulations should be low for gold. Counting the electrons involved in the free and upper d-bands, we could expect the ratio $\mathrm{R}_{\mathrm{bf}}=\mathrm{n}_{0}{ }_{\mathrm{f}}^{\mathrm{f}} / \mathrm{n}_{0} \mathrm{~b}$ to lie around 0.5 , even if values down to 0.1 may be acceptable if all valence $\mathrm{d}$ electrons are considered as a whole. However, $\mathrm{n}_{0}{ }^{\mathrm{b}}$ is also influenced by the actual joint density of states participating in the interband transitions; thus, a value smaller than $\mathrm{n}_{0}{ }^{\mathrm{f}}$ may also be considered. In addition, we use the bulk polarizabilities throughout the calculations, including in the selvedge. It may be considered that, in this region, the polarizabilities differ from the bulk as a consequence of the change in the atomic coordination in the topmost layers. In particular, we could expect an increase in the bound electron polarizability due to lower constraints towards the air side than towards the bulk gold side. Reversely, for the topmost layer of gold atoms, their free electrons are involved in the Au-S bond formation, which leads to a dramatic decrease in the free electron polarizability in this region. In addition, the infrared free electron polarizability may be overestimated as a result of using a simple Drude model..$^{5,17,18}$ This could also impact on the balance between free and bound electron terms in the total nonlinear surface susceptibility. As a consequence, in the interface region, neither the actual electron densities nor the polarizabilities are accurately determined. In order to account for this situation, and as only the bulk products $\mathrm{n}_{0}{ }^{\mathrm{f}} \alpha^{\mathrm{f}}$ and $\mathrm{n}_{0}{ }^{\mathrm{b}} \alpha^{\mathrm{b}}$ are known from the dielectric function, tuning parameters $n_{0} f / b$ is an easy way to modulate the effective polarizabilities of each family of electrons while keeping the products constant. As a consequence, in order to get a broad screening of the line shapes of the amplitudes and phases of the simulated full effective nonlinear susceptibilities under the three schemes, we have chosen to screen the values of $\mathrm{R}_{\mathrm{bf}}$ over a wide interval between 0.1 and 40 . In this frame, the contribution from the free electrons is analogous to silver and will serve as the regular contribution for gold. The interband contributions on the other hand constitute the corrective terms.

For schemes 1 and 2 (Fig. 2), the free electron contribution is so high (comparing to all others) that changing the value of $\mathrm{R}_{\mathrm{bf}}$ in the range $[0.1 ; 2]$ has almost no impact on the final result. However, for $\mathrm{R}_{\mathrm{bf}}$ above 5, the interband contribution starts emerging from the free electron one and a transition to a new regime shows up in two ways: the rise of a double peak in the central region of the amplitude spectra and a sign change at the high energy end. We checked that $\varepsilon_{2}{ }^{b}$ has no influence on the spectra, as expected. ${ }^{2}$ We note that the slope of the growing amplitude of the free electron background (from blue to red) remains bigger than for the experimental data and that all phase curves converge at $700 \mathrm{~nm}$, as was expected from the analysis of the individual contributions.

Figure 3 shows the effective nonlinear susceptibilities of gold for scheme 3 , for which a dependency in $\varepsilon_{2}{ }^{b}$ is observed. The effect of both parameters $\varepsilon_{2}{ }^{b}$ and $R_{b f}$ appears in panels (a), (c) and (b), (d), respectively. Increasing either of them changes the balance between free and bound electron contribution in favor of the latter. From the previous analysis, we know that the zzz term remains rather flat, the maxima therefore come from the peaks in the free electron xxz, xzx, and bulk terms, and from the interband bulk term. The former favor a maximum in the $600 \mathrm{~nm}$ range, whereas the latter induces a peak around $500 \mathrm{~nm}$. The same phenomenon happens for the phases, which remain constant at $700 \mathrm{~nm}$ for the reasons explained above and increase at $400 \mathrm{~nm}$ towards a value depending on the balance between the free electron (low phase) and bound electron (high phase) contributions. Finally, when the bound electron terms are extremely favored [e.g., for high $\mathrm{R}_{\mathrm{bf}}$ in panel (d)], the overall effective susceptibility changes sign at $700 \mathrm{~nm}$ following the phase of the interband zzz term.

We may now compare the simulations above to the experimental data, ${ }^{1}$ refer to Fig. 4 , which give access to the line shape of the amplitude and absolute value of the phase of the nonlinear effective susceptibility of a thiolated gold surface as a function of the visible wavelength. In order to find the best fit, we use a scaling factor $\mathrm{S}$ for the total effective nonlinear susceptibility of gold, leading to a simulation function [Eq. (A7)]

$$
\chi_{\text {sim }}^{\text {eff }}=S\left[\chi_{\text {gold }}^{\text {eff, }}+R_{b f} \chi_{\text {gold }}^{\text {eff,b }}\right]
$$

We then choose the best values for parameters $S, R_{b f}$, and $\varepsilon_{2}{ }^{b}$, leading to the simulations shown in Fig. 4 for schemes 

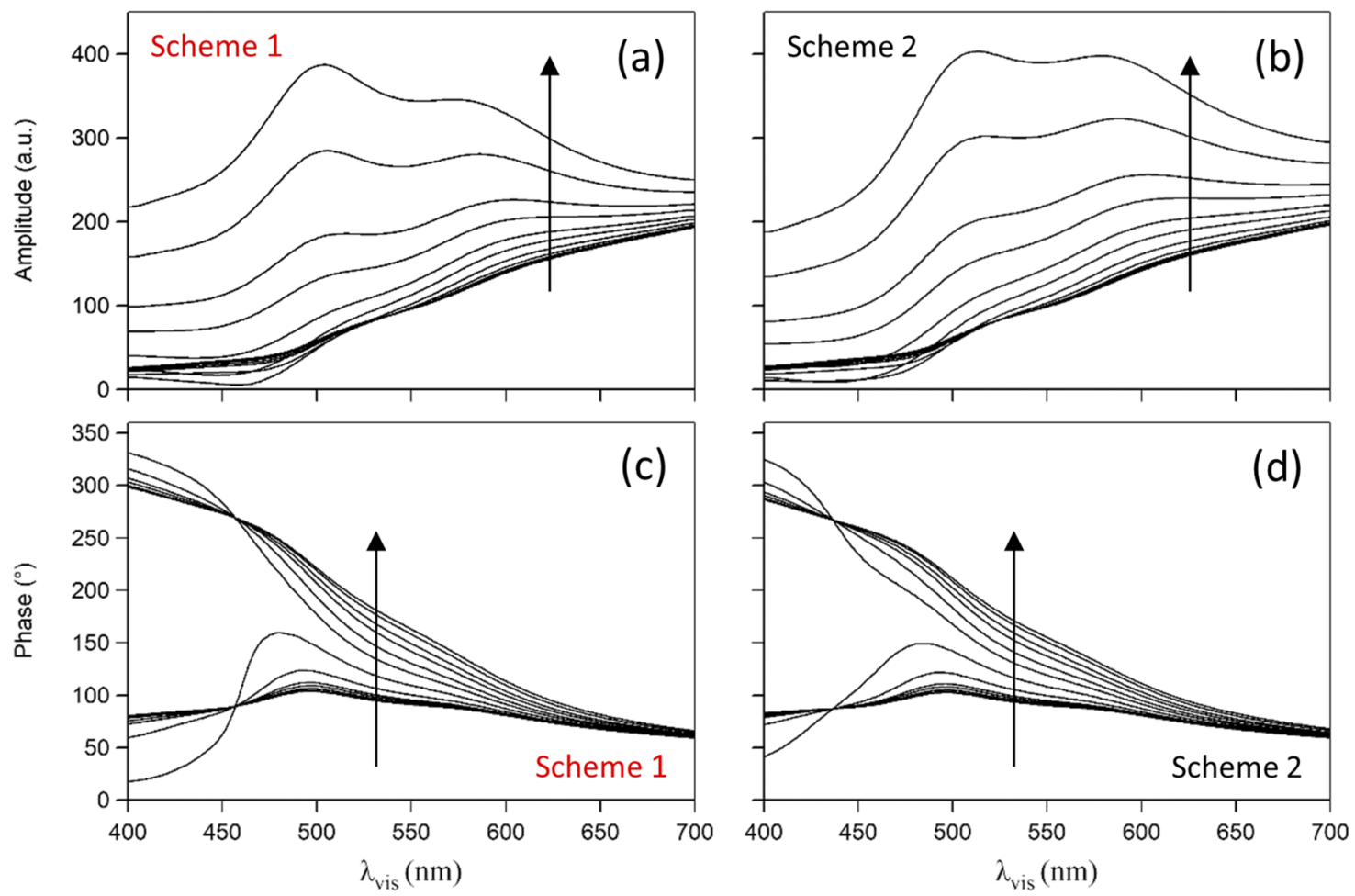

FIG. 2. Amplitudes [(a) and (b)] and phases [(c) and (d)] of the simulated effective nonlinear susceptibility of gold versus the visible wavelength $\left(\lambda_{\mathrm{vis}}\right)$ according to schemes 1 [(a) and (c)] and 2 [(b) and (d)] for various values of $\mathrm{R}_{\mathrm{bf}}$ from 0.1 to 40 , increasing along the arrows. Infrared wavenumber is fixed at $2900 \mathrm{~cm}^{-1}$ and $\varepsilon_{2}{ }^{\mathrm{b}}=1.5$.

$1-3$. We clearly see that the three schemes account for the trends in experimental data, even if some discrepancies remain.
Schemes 1 and 2 give equivalent variations for the phase curve, for which agreement with experiment is very good at high energy (below $560 \mathrm{~nm}$ ). Scheme 2 provides a better fit
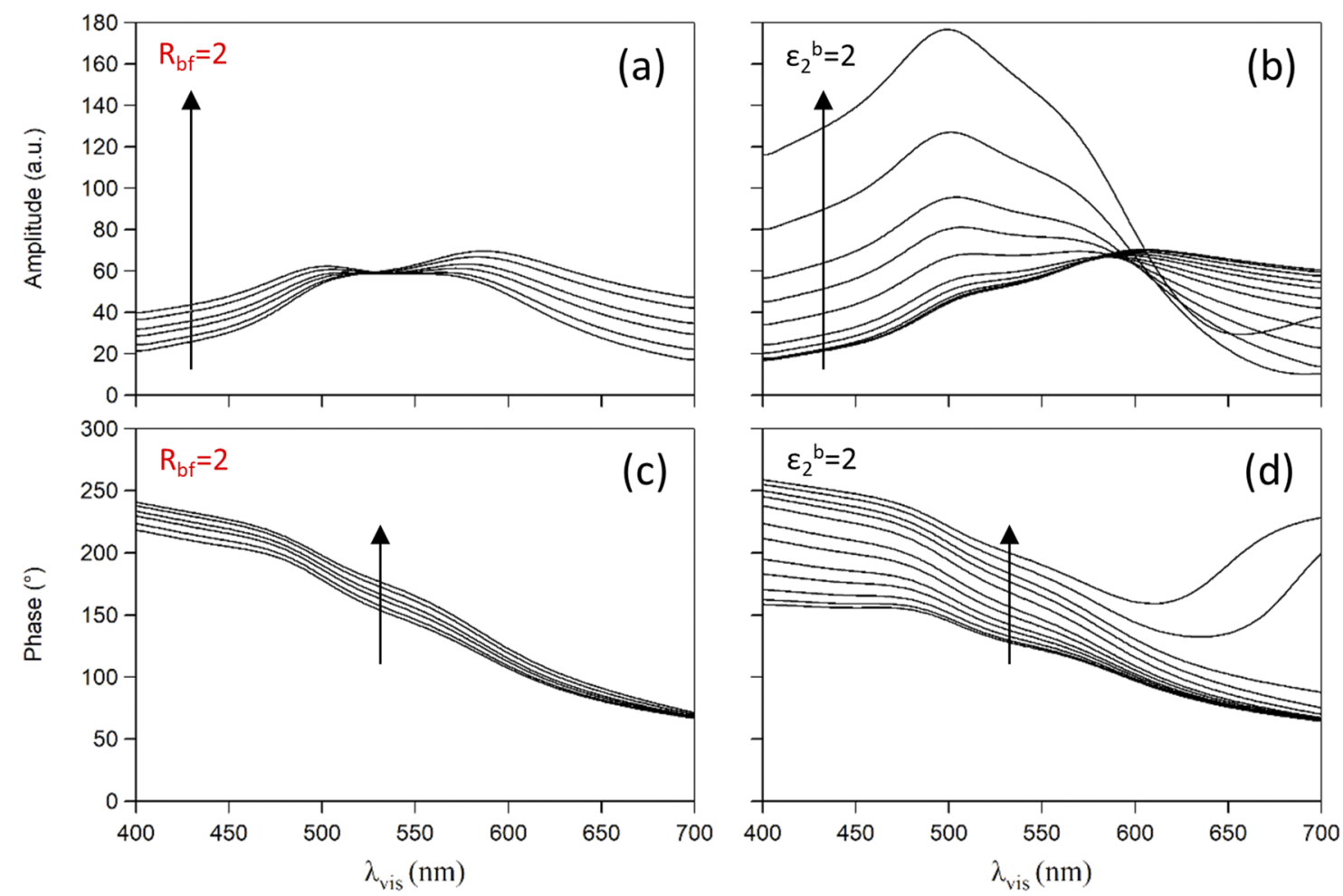

FIG. 3. Evolution of the amplitudes [(a) and (b)] and phases [(c) and (d)] of the simulated effective nonlinear susceptibility of gold for scheme 3 with parameter $\varepsilon_{2}{ }^{b}$ increasing along the arrows from 1.5 to 8 with $R_{b f}=2$ [(a) and (c)] and parameter $R_{b f}$ increasing from 0.1 to 10 with $\varepsilon_{2}{ }^{b}=2$ [(b) and (d)]. Infrared wavenumber is fixed at $2900 \mathrm{~cm}^{-1}$. 

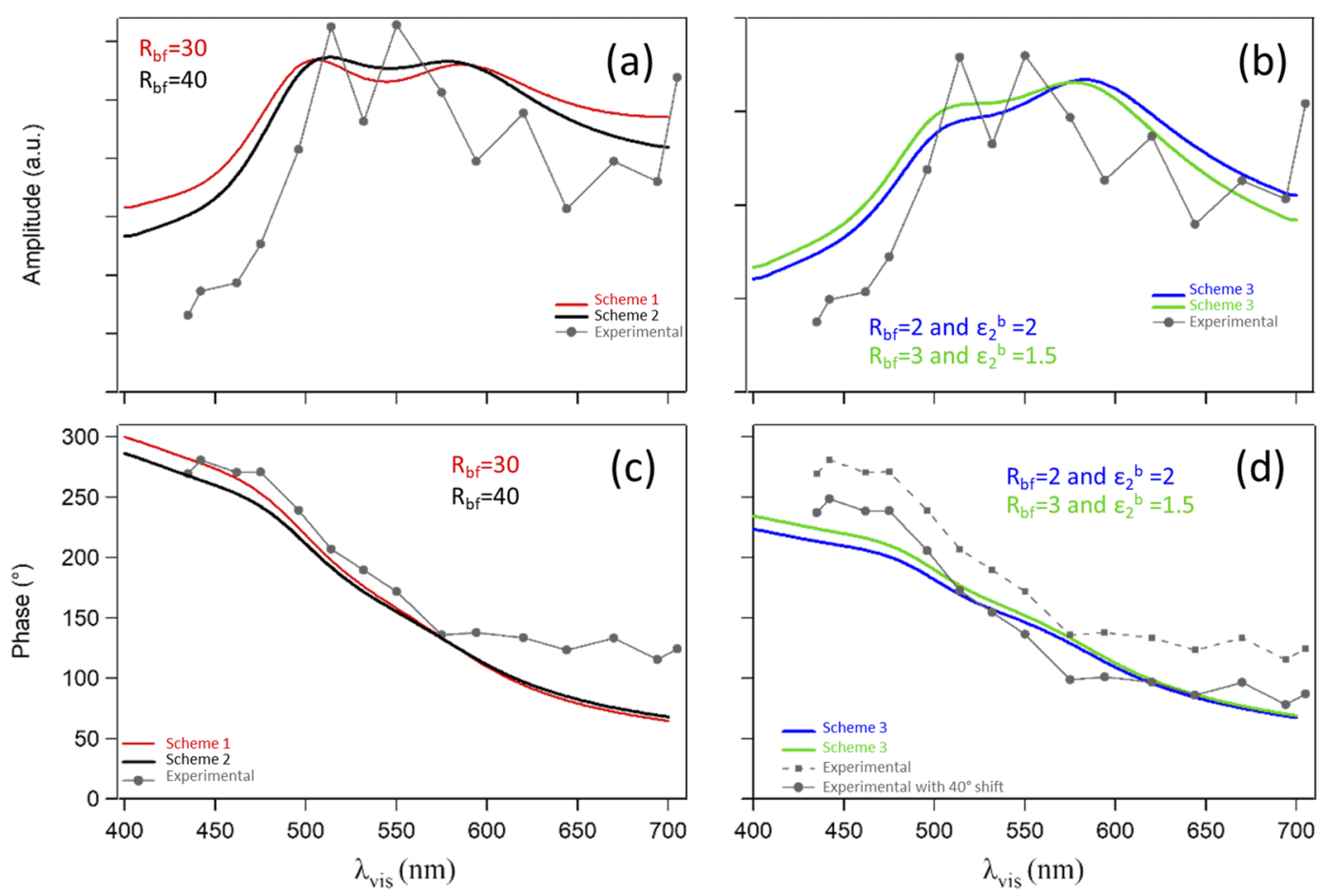

FIG. 4. Comparison of experimental (dots and squares) and simulated effective susceptibilities of gold for schemes 1 (red, $\left.R_{b f}=30\right), 2$ (black, $\left.R_{b f}=40\right)[(a)$ and (c)], and 3 [(b) and (d)] in amplitude [(a) and (b)] and phase [(c) and (d)]. The experimental points are reproduced with permission from Dalstein et al., J. Chem. Phys. 148, 134701 (2018). Simulated amplitudes are rescaled to match the experimental data. In (b) and (d), the parameters are $R_{b f}=2$ and $\varepsilon_{2}{ }^{b}=2$ (blue), and $\mathrm{R}_{\mathrm{bf}}=3$ and $\varepsilon_{2}{ }^{\mathrm{b}}=1.5$ (green). The original experimental phase curve (squares) in (d) is shifted down by $40^{\circ}$ (dots).

in amplitude, as the central peak is more pronounced than for scheme 1. As for scheme 3, which depends on $\mathrm{R}_{\mathrm{bf}}$ and $\varepsilon_{2}{ }^{\mathrm{b}}$, we have found several parameter sets which lead to a reasonable fit in amplitude, for which both $R_{b f}$ and $\varepsilon_{2}{ }^{b}$ range from 1.5 to 3 . The best ones are displayed in Fig. 4. The low values of $\varepsilon_{2}{ }^{\mathrm{b}}$ agree with the simple view of a very weak polarizability for bound electrons in the infrared. It is tempting to choose the scheme 3 as the best one to interpret the experimental data. However, even if the simulated phase follows a correct trend, as illustrated in Fig. 4(d), its absolute value is around $40^{\circ}$ lower than the experimental one. In addition, the three schemes underestimate the phase at $700 \mathrm{~nm}$.

The most surprising result from these simulations is the high values required for parameter $R_{b f}$. For schemes 1 and 2 , such high values are needed in order to recover the line shape of the amplitude (30 and 40, respectively). Even for scheme $3, R_{b f}$ has a closer value to the predicted one (0.5), but still needs to be adjusted four to six times higher (corresponding to an effective bound electron density lower than expected). It looks like the free electron contribution was calculated too high and the bound electron one underestimated. From scheme 1 to scheme 3 , the mutual screening between both contributions evolves from fully unscreened free electron to fully unscreened bound electron terms. Comparison with the corresponding values of $\mathrm{R}_{\mathrm{bf}}$ suggests that screening of the bound electron term is too high, as it cannot compete enough with the free electron contributions. However, it is not possible to consider a model for which the screening of the bound electron terms would be lower than for scheme 3 . This means that the free electron terms are rather systematically evaluated too high. This may be due to the fact that we use the bulk polarizabilities throughout the calculations, including in the selvedge. In this region, the free and bound electron polarizabilities may differ from the bulk as a consequence of the change in the atomic coordination in the topmost layer.

The model is flexible enough to allow for some additional fine tuning of the hypotheses chosen for the simulations. For example, it makes it possible to decrease the value of the free electron polarizability in the interface region only (i.e., for the surface but not the bulk terms) in order to account for the fact that the free electrons of the first gold layer are involved in the Au-S bonds, while still controlling the balance between free and bound electrons through the $\mathrm{R}_{\mathrm{bf}}$ factor. We have also performed simulations under these assumptions, with the ratio of surface to bulk free electron density as an additional free parameter. A variety of line shapes for the amplitudes and phases may be obtained for several parameter sets, but the overall dependence on the electron density ratios follows the same trend as in Fig. 4 and shows no new phenomenon.

The choice of parameters and remaining discrepancies between the simulations and the experimental data are discussed below, but at this stage, we note that the model developed in this work allows us to recover the trends of the experimental curves with only one (schemes 1 and 2) or two (scheme 3) free parameters, without the need of a resonance with a surface state.

\section{THE EFFECTIVE SURFACE STATE SCENARIO}

We now turn to the electronic surface state scenario, modeled by a nonlinear surface susceptibility $\chi_{D}$. Since the 
existence of such a surface state is postulated at this stage, the parameters must be determined by curve fitting to determine its properties. There are several possibilities for the choice of a fitting function: considering a Lorentzian profile, the SFG process may become resonant with either the visible or the SFG beam (single Lorentzian profile), a combination of both (double Lorentzian profile) or with both beams simultaneously (doubly resonant process). As explained in Appendix B, the doubly resonant scheme is not favored because the infrared photon may not induce a resonant transition inside a single electronic state. The most general form for the resonant susceptibility is therefore a sum of two Lorentzian functions, resonant with the visible and SFG photons, respectively.

We first performed the same analysis as in Ref. 6 as reported in the supplementary material. However, it has two drawbacks. First, the constant nonresonant term in the supplementary material, Eq. (S1), which seems essential for the correct rendering of the experimental amplitudes, has no physical meaning. It has been introduced by analogy to the usual data analysis of vibrational SFG spectra. In this case, however, this term originates in the substrate contribution, usually of an electronic origin, and indeed not resonant when scanning the infrared frequency. In our case, this term is introduced in addition to the resonant surface state. It therefore stems from the other gold contributions to the SFG signals and, as we have seen in Sec. III, those are constant neither in amplitude nor in phase. Second, the experimental data represent the effective susceptibility of gold, which means that they relate to the incoming electric fields through Fresnel factors. Again, we have shown that these factors vary in amplitude and in phase with the visible wavelength. ${ }^{1,2}$ In order to improve these fits, we investigate the possibility to account for the experimental data using a sum of two contributions: one from the nonlinear properties of gold as calculated in Sec. III and the other from the resonance with a surface state, accounting for Fresnel reflectivity (i.e., through an effective surface nonlinear susceptibility). The effective fitting function is

$$
\begin{aligned}
\chi_{\text {fit }}^{\text {eff }}= & A \chi_{\text {gold }}^{\text {eff }}\left(\mathrm{R}_{\mathrm{bf}}=0.4\right)+\chi_{\mathrm{D}}^{\mathrm{eff}}=A \chi_{\mathrm{gold}}^{\mathrm{eff}}(0.4) \\
& +\mathrm{F}_{\mathrm{vis}} \frac{\mathrm{Be}^{\mathrm{i} \varphi_{\text {vis }}}}{\omega_{\text {vis }}-\omega_{\mathrm{SS}}+\mathrm{i} \Gamma_{\mathrm{SS}}}+\mathrm{F}_{\mathrm{SFG}} \frac{\mathrm{Ce}^{\mathrm{i} \varphi_{\mathrm{SFG}}}}{\omega_{\mathrm{SFG}}-\omega_{\mathrm{SS}}+\mathrm{i} \Gamma_{\mathrm{SS}}},
\end{aligned}
$$

with eight unknown parameters in the general case. Having explicitly factored, the Fresnel contributions legitimate here the use of constant amplitudes and phases. The first term in the sum refers to the calculations of Sec. III for the nonlinear response of gold. It has no free parameter, except for a scaling factor $A$, and the $\mathrm{R}_{\mathrm{bf}}$ ratio. As explained earlier, this ratio is supposed to remain close to 0.5 . In order to account for the fact that some bound electrons from lower energy electronic states additionally screen the bound electron response, we slightly decrease the $R_{b f}$ value in this part to 0.4 . With such a value, this A-term embeds the contribution from the bound electrons, but it originates essentially from the free electrons. As such, it represents the silver-like regular contribution for gold. Conversely, in this effective surface state scenario, the
$\mathrm{B}$ and $\mathrm{C}$ terms account for the resonant corrective part, specific to gold. Under the previous assumptions, some amplitude coefficients (A, B, or C) may vanish. In addition, there may be some relationship between the phases and/or the amplitudes: under the approximation of high energies discussed in Appendix B, amplitude coefficients B and C may be close to complex conjugate numbers.

As a matter of fact, we can check that an accurate fitting of the experimental points is possible with such a function. We fit the complex function deduced from the experiment (refer to Fig. 4) by simultaneously fitting the real and imaginary parts using a single parameter set. This avoids the risk to obtain multiple fitting parameter sets, as is the case for the general fit of SFG spectra ${ }^{19}$ (i.e., without any phase information). In order to fit over the widest range of visible wavelengths, we add to our data one point at $401 \mathrm{~nm}$ taken from Ref. 6. We acknowledge the fact that the additional constraint due to the presence of this point makes convergence of the fits more difficult, but the result is more accurate. It also allows us to discard some parameter sets for which fitting is fine except for this point. We understand that it is more constraining to fit the shape of our experimental data than in Ref. 6, as we have fifteen experimental points instead of four.

Depending on the origin of the surface state, there are two possibilities for the additional term $\chi^{\text {eff }}$. If the surface state is intrinsic to gold, then its associated surface nonlinear polarization is located inside the metal, where the electric fields have to be evaluated. On the contrary, if it relates to the adsorption process and the Au-S bond, it may rather be considered as external to gold, in which case the associated polarization and the electric fields have to be evaluated outside the metal. As mentioned in Ref. 2, for a given source of nonlinear polarization, several different nonlinear susceptibilities may formally be defined (depending on which medium the electric fields and nonlinear polarization are evaluated), all of them eventually leading to the same effective susceptibility and far field measurements. The situation is different here, as the effective susceptibility of the surface state itself may take two values, in accordance with the medium wherein the nonlinear process actually takes place. Under the formalism used throughout this study, the Fresnel factors and their dispersions for the external ${ }^{1}$ and internal ${ }^{2}$ cases have been studied before.

As there are four nonvanishing tensor components at the isotropic surface, we have to make some assumptions in order to fit our data according to Eq. (5). We first assume that the transition dipole moment related to the excitation of the surface state is either parallel or perpendicular to the surface plane (i.e., along $\mathrm{x}$ or $\mathrm{z}$, respectively, according to Fig. 1), as both have been described in the literature. ${ }^{10,12} \mathrm{~A}$ general case shall correspond to a linear combination of both. In the parallel case, the terms resonant with $\omega_{3}$ are xxz and xzx, and with $\omega_{1}$ are $\mathrm{xxz}$ and zxx. In the perpendicular case, we have zxx and zzz as resonant terms with $\omega_{3}$, and Xzx and zzz with $\omega_{1}$. In order to select the relevant terms, we recall that the dominant Fresnel factors for a nonlinear polarization inside gold are zxx and Xzx, whereas for a polarization outside the metal they are zzz and xxz. Table I shows the main relevant combinations of terms in both cases. We therefore consider four tensor combinations 
TABLE I. Choice of relevant nonlinear susceptibility tensor components for the surface state in Eq. (6).

\begin{tabular}{lcccc}
\hline \hline Transition dipole & \multicolumn{2}{c}{ Along x } & \multicolumn{2}{c}{ Along $\mathrm{z}$} \\
\hline Resonance with beam & $\omega_{3}\left(\mathrm{~F}_{\mathrm{SFG}}\right)$ & $\omega_{1}\left(\mathrm{~F}_{\mathrm{vis}}\right)$ & $\omega_{3}\left(\mathrm{~F}_{\mathrm{SFG}}\right)$ & $\omega_{1}\left(\mathrm{~F}_{\mathrm{vis}}\right)$ \\
Inside gold & $\mathrm{xzx}$ & $\mathrm{zxx}$ & $\mathrm{zxx}$ & $\mathrm{xzx}$ \\
Outside gold & $\mathrm{xxz}$ & $\mathrm{xxz}$ & $\mathrm{zzz}$ & $\mathrm{zzz}$ \\
\hline \hline
\end{tabular}

in the following, coupled to a choice of three possible $\chi^{\mathrm{eff}}{ }_{\text {gold }}$ (i.e., schemes 1-3). Only schemes 1 and 3 are considered for actual fitting as scheme 2 does not significantly differ from scheme 1 for $R_{b f}=0.4$, which leads to eight combinations for the full effective fit function.

Among all the parameter sets found by curve fitting using Eq. (5), without any constraints imposed on the parameters, we have first selected the nine parameters showing the best agreement both in amplitude and in phase. They span six of the eight combinations above. Figure 5 shows the best fit for each combination, with the parameters listed in Table II. We have checked that the properties of the fitting functions based on the three remaining parameter sets do not differ from the six selected ones. The fits are of lesser quality than in Fig. S1, supplementary material, in particular, for the phases at high energy, the main problem being that the phases now have to follow the evolutions of the Fresnel factors and of the gold terms. However, the curves prove that such a fit may account for the experimental data, in a more rigorous way than in Fig. $\mathrm{S} 1$, supplementary material. There is no overall coherence on most of the parameters: some sets show Lorentzian amplitudes close to each other (ESS1, ESS3, ESS5, and ESS6) and some exhibit a phase relationship between the two Lorentzians $\left(180^{\circ}\right.$ phase shift for ESS1, complex conjugate for ESS3, in phase for ESS5). As for the characteristics of the surface state, they also span a wide range of energies [between 11600 and $19800 \mathrm{~cm}^{-1}(1.44$ and $2.46 \mathrm{eV})$, lower than in Table S1, supplementary material] and widths (between 1200 and $4400 \mathrm{~cm}^{-1}$ ). As a consequence, the analysis does not allow at this stage to choose the most probable combination for the gold term, the location of the surface state, and the direction of its transition dipole moment. We note that the curve (ESS3) relates to Lorentzian resonances for which the approximation of the high energies holds ( $\mathrm{B}$ and $\mathrm{C}$ are close to complex conjugates).

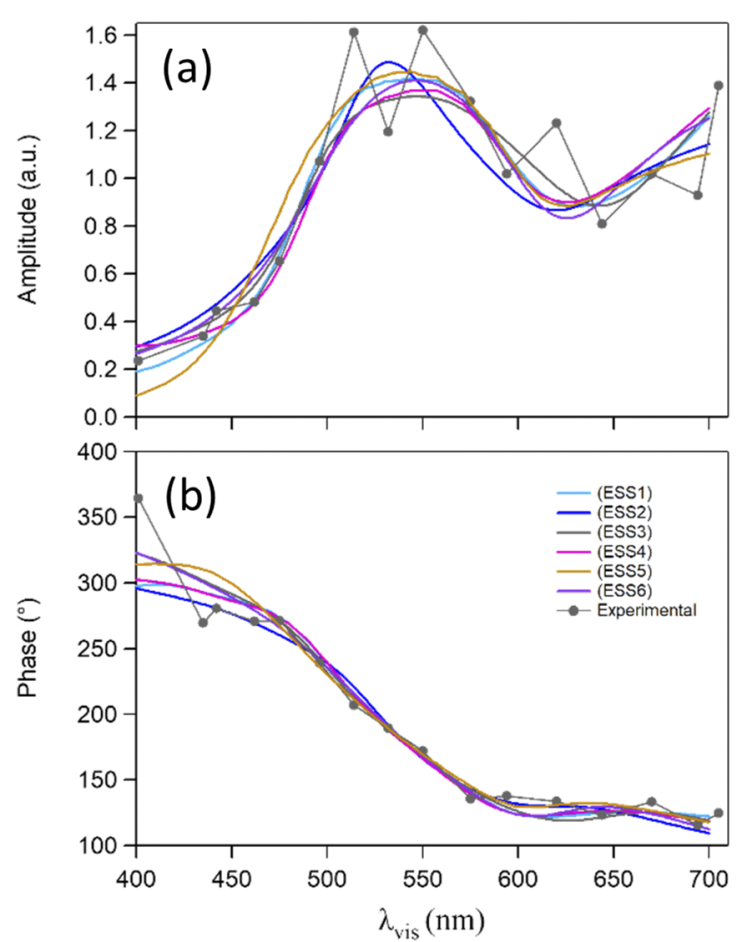

FIG. 5. Fit of the experimental effective nonlinear susceptibility of gold along Eq. (5) in amplitude (a) and in phase (b). The parameters for each curve may be found in Table II.

\section{DISCUSSION}

\section{A. Comparison between scenarios}

The analysis above proves that it is possible to account for the major features of the experimental data under both interband and effective surface state hypotheses. In the interband scenario, the agreement between theory and simulation is only partial, but there is only one free parameter to the model. For the effective surface state scenario, a much better agreement is obtained but most parameters stem from a direct curve fitting. On the other hand, the first case is self-consistent, while the second one still requires some analysis of the nature of the surface states involved.

Simulations under the interband scenario show two peaks in amplitude roughly corresponding to the interband excitation at the visible and SFG frequencies. This double peak is not clearly seen in the experimental data. In the effective

TABLE II. Fit parameters for the curves in Fig. 5.

\begin{tabular}{|c|c|c|c|c|c|c|}
\hline Curves & ESS1 & ESS2 & ESS3 & ESS4 & ESS5 & ESS6 \\
\hline A-term & Scheme 1 & Scheme 1 & Scheme 3 & Scheme 3 & Scheme 3 & Scheme 3 \\
\hline B-term & $\mathrm{F}_{\mathrm{XZX}}$ (int.) & $\mathrm{F}_{\mathrm{ZzZ}}($ ext.) & $\mathrm{F}_{\mathrm{XZX}}$ (int.) & $\mathrm{F}_{\mathrm{Zxx}}$ (int.) & $\mathrm{F}_{\mathrm{zzz}}$ (ext.) & $F_{X x z}$ (ext.) \\
\hline C-term & $\mathrm{F}_{\mathrm{Zxx}}$ (int.) & $\mathrm{F}_{\mathrm{zzz}}$ (ext.) & $\mathrm{F}_{\mathrm{zXX}}$ (int.) & $\mathrm{F}_{\mathrm{XzX}}$ (int.) & $\mathrm{F}_{\mathrm{zzz}}$ (ext.) & $F_{x x z}$ (ext.) \\
\hline A & $2.54 \cdot 10^{-3}$ & $5.24 \cdot 10^{-3}$ & $2.95 \cdot 10^{-2}$ & $3.88 \cdot 10^{-2}$ & $5.50 \cdot 10^{-2}$ & $2.79 \cdot 10^{-2}$ \\
\hline B (vis) & $2.469 \cdot 10^{6}$ & 3526 & $7.20510^{5}$ & $9.63610^{5}$ & 5601 & $1.321 \cdot 10^{4}$ \\
\hline $\mathrm{C}(\mathrm{SFG})$ & $2.331 \cdot 10^{6}$ & 769.8 & $8.931 \cdot 10^{5}$ & $2.848 \cdot 10^{6}$ & 3860 & $1.363 \cdot 10^{4}$ \\
\hline$\omega(\mathrm{SS})\left(\mathrm{cm}^{-1}\right)$ & 11604.5 & 19132.3 & 17084.0 & 14570.6 & 19817.6 & 18798.8 \\
\hline$\Gamma(\mathrm{SS})\left(\mathrm{cm}^{-1}\right)$ & 1213.4 & 1419.7 & 2496.8 & 4396.0 & 1645.9 & 2472.7 \\
\hline$\varphi($ vis $)(\mathrm{deg})$ & 120.4 & 247.5 & 138.9 & 317.6 & 318.1 & 11.3 \\
\hline$\varphi(\mathrm{SFG})(\mathrm{deg})$ & 297.1 & 319.9 & 228.9 & 163.4 & 324.4 & 105.4 \\
\hline
\end{tabular}


surface state scenario, the double peak is also present, but curve fitting, with an appropriate choice of parameters, allows the interference between them to artificially show up as a single peak. It is difficult to know whether this is an artifact of fitting or whether it represents an actual phenomenon; this illustrates the extreme flexibility of the surface state scenario.

\section{B. Selection of the best scenarios}

We may refine the analysis using again the comparison between gold and silver. We know that the SFG response of silver is experimentally much lower than for gold. As the simulations for silver lead essentially to the same SFG intensities [or even higher, see Fig. 6(b)] as for gold under the same assumptions (i.e., regular contributions only), this gives the evidence that the corrective terms specific to gold must exceed the regular terms by a large amount.

In Fig. 6(a), we calculate the amplitude ratios of these contributions for the effective surface state scenario. We clearly see that only curve ESS1, and to a lower extent curve ESS2, meets the criterion. The other parameter sets are discarded because the amplitudes of the regular and corrective terms match, and the final dispersion line shape they produce for the total effective susceptibility of gold solely originates through an interference process. In Fig. 6(b), we separate and compare the calculated SFG intensities for the interband (schemes 1-3) and effective surface state scenarios (curves ESS1 and ESS2) between regular and corrective terms. In the same way as above, the former are too high for scheme 3 in the interband

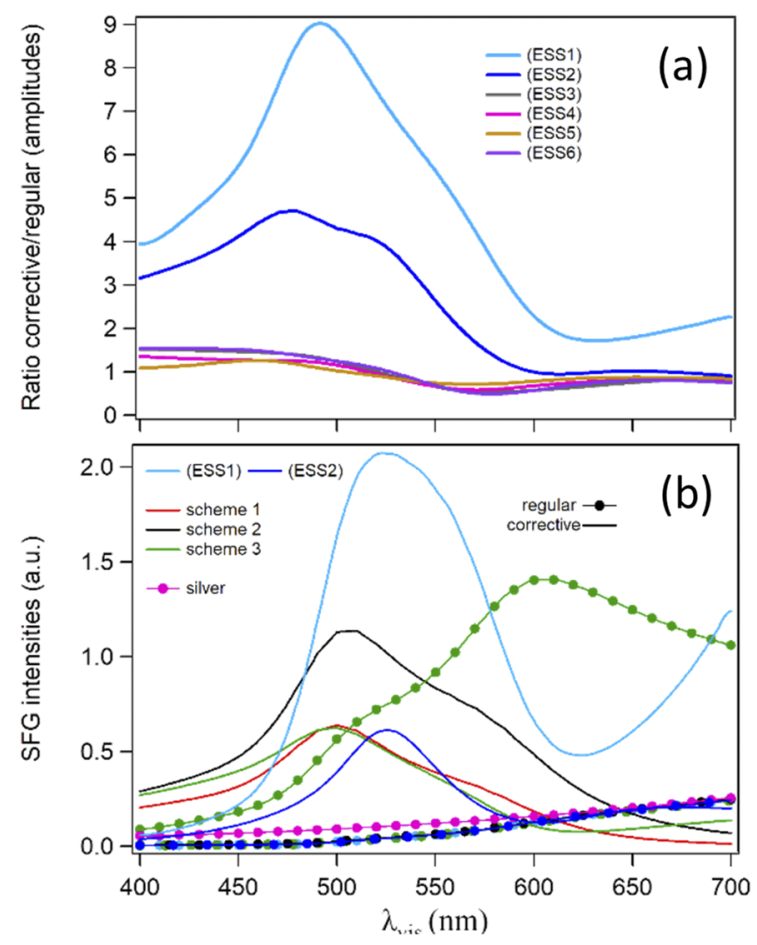

FIG. 6. (a) Ratios of the amplitudes of the surface state contributions [B and C-terms in Eq. (5)] to the regular A-terms for the six curves shown in Fig. 5. (b) Comparison of the SFG regular and corrective intensities. Scaling is common to all regular curves, except for scheme 3 (scaling for the corrective curve is common to scheme 1). scenario, which is not compatible with the comparison with silver.

As a consequence, we finally retain only schemes 1 and 2 with high $\mathrm{R}_{\mathrm{bf}}$ in the interband scenario, and curve ESS1 in the effective surface state scenario, for which (Table II) the regular contribution also follows from scheme 1 (or equivalently scheme 2). The comparison between gold and silver allows us to exclude the hypothesis of scheme 3 for gold (i.e., spill out of the bound electrons), and the same conclusion has also been drawn before for silver itself. ${ }^{2}$ The two remaining schemes are compatible with a conventional picture of the free electrons at the interface, either spilling out or overlapping in space with the bound electrons.

The interband and surface state scenarios each have their advantages and drawbacks. As noted above, schemes 1 and 2 in the interband hypothesis need a strong adjustment of coefficient $R_{b f}$ in order to account for the respective weights of interband and free electron terms. In addition, the phase evolution shows some discrepancies with experimental data at low energy, and the simulated amplitude at high energy is still too high. This leads us to question the accuracy of the experimental values. A fitting procedure was originally used to analyze the vibrational spectra, ${ }^{1}$ which intrinsically leads to rather large error bars on the parameters. ${ }^{19}$ As for the phases, it has been shown ${ }^{20}$ that very similar fitting curves could be obtained within a $30^{\circ}$ interval, which constitute an intrinsic error bar after experimental data analysis. Some approximations may also distort the values of the parameters: as for the absolute phases, for example, their origins stem from the vanishing phase of vibration modes in the harmonic oscillator approximation, which is usually not questioned for methyl stretching modes in alkanes. In addition, we used an average molecular Fresnel factor in order to compare the relative intensities of symmetric and antisymmetric stretches. However, even if its phase spans $\pm 15^{\circ}$ on the blue side of the spectrum as a function of the tilt angle, this has no influence on the calculated phase at $700 \mathrm{~nm}$, as all curves converge to the same values (see Fig. 2 in Ref. 1). The phase being very sensitive to the Fresnel factors, we may also question the use of a real index of refraction for the molecular monolayer in the infrared. As a matter of fact, the molecules absorb light in the probed infrared region, so there should be an absorptive part. For alkane chains, we find in the literature an imaginary refractive index of 0.17 or less. ${ }^{21,22}$ This value leads to a phase shift of $-16^{\circ}$, still too low to explain to observed figures, but within the range of errors obtainable from the fitting procedure.

In the effective surface state hypothesis, the choice of curve ESS1 leads to interesting conclusions: the nonlinear polarization resonant with the surface state is evaluated inside gold and is therefore intrinsic to the surface properties of the metal, not of the adsorbate or adsorbate-metal bond. In addition, its transition dipole moment lies preferentially along the metal surface, as in Ref. 10. This gives us some properties for this state which we may compare to the known surface states and transition from the literature.

\section{Origin of the surface state transition}

The theoretical and experimental literature on surface states in metals is abundant, ${ }^{7,8}$ and this topic is still an active 
field of research. ${ }^{23,24}$ They have to be taken into account as far as surface processes are concerned since, for example, $22 \%$ of the electrons in the surface layer of $\operatorname{Ag}(100)$ are found in surface states or resonances. ${ }^{25}$ Intrinsic surface states appear as special solutions of the Schrödinger equations for electrons inside the metal, which, unlike the bulk solutions, exponentially decay on both sides of the interface between the metal and the exterior, thus maximizing their wavefunction at the vicinity of the surface. ${ }^{7}$ They are often qualified as Shockley and Tamm states, depending on their sp or d character, respectively. ${ }^{26}$ Dispersion of the surface states depends on the crystalline orientation at the surface and may be displayed in a projection of the bulk band structure onto the corresponding surface Brillouin zone, as a relationship between energy and wave vector parallel to the surface. Their energy-wavevector relationships impose that their energies lie inside the gaps between the projected bulk bands, but they may also be degenerated with bulk states, in which case they couple to them and become surface resonances ${ }^{27}$ (i.e., a particular bulk state with enhanced wavefunction at the interface). In addition, the intrinsic surface states are perturbed upon molecular adsorption at the surface, whereas this phenomenon also gives rise to new extrinsic surface states through hybridization between metal electrons and molecular orbitals. ${ }^{28}$ Finally, as a non-zero electronic density outside the metal creates an image charge inside the conduction electron gas of the metal, the Coulomb interaction between the two charges may create new bound states, usually above the Fermi level, called image states. ${ }^{28}$ Experimentally, all these states may be investigated on single crystal surfaces by photo-emission and inverse photo-emission spectroscopies for occupied and empty states, respectively. ${ }^{29}$ Other experimental methods include electroreflectance spectroscopy $^{30}$ and reflection anisotropy spectroscopy (RAS). ${ }^{31}$ Several publications mention specific transitions between two such states which may cause signal enhancements in SHG and SFG. ${ }^{6,10-12}$ However, these publications do not consider the interband scenario to account for their data, although Urbach et al. ${ }^{10}$ separately detect the interband transition peak on silver.

Interpretation of our results in terms of surfaces states is essentially a matter of finding a resonant transition between two states at the surface of gold, compatible with the expected structure of the surface in our case, namely, polycrystalline with a preferred (111) faceted orientation. This looks rather paradoxical as surface states relate by essence to the high degree of crystallinity of the interface and usually show up on single crystals. For polycrystalline samples like ours, surface states should be damped or smeared out as a consequence of the small crystallite sizes and mismatch at their boundaries. In addition, the high level of SFG intensities on gold in the blue-green region of the visible spectrum have been experimentally observed for at least four surface orientations, namely, (111), (110), (100), and (210), ${ }^{32}$ which supposes that there should be a dedicated surface state transition for all of them. However, it turns out that experimental evidence by both direct and inverse photoemission and theoretical calculations show that surface transitions in the 2-3 eV energy range seem possible, at least on the low index (111), (110), and (100) crystals.
On $\mathrm{Au}(111)$, a Shockley surface state with a parabolic dispersion is well documented at the $\bar{\Gamma}$ point of the projected Brillouin zone, ${ }^{33,34}$ with a minimum located $0.4 \mathrm{eV}$ below the Fermi level $\left(\mathrm{E}_{\mathrm{F}}\right)$. However, there is no empty state at this point allowing a vertical transition from there in the visible energy range. Image states have been evidenced at $\bar{\Gamma}$, but they lie too high (around $4 \mathrm{eV}$ above $\mathrm{E}_{\mathrm{F}}$ ) to be accessible. ${ }^{35}$ However, calculations ${ }^{36}$ predict the possibility of a transition at $\overline{\mathrm{M}}$ point, which is supposed to be responsible for the observed enhancements of SHG signals at 2.5 and $1.25 \mathrm{eV}^{11,37}$ This transition relies on a Tamm state located between 1.4 and $1.7 \mathrm{eV}$ below the Fermi level ${ }^{24,38}$ and an empty state between 1.3 and $1.7 \mathrm{eV}$ above it. ${ }^{36,38}$ Although the energy difference remains higher than the experimental value, coupling of the occupied state (described as a surface resonance instead of a true surface state ${ }^{24,38}$ ) to bulk bands at $\mathrm{L}$ and $\mathrm{X}$ points, where bulk interband transitions occur, may account for the experimental evidence. On $\mathrm{Au}(110)$, the existence of surface states is predicted at $\bar{X}$ [along (110)] and $\bar{Y}$ [along (001)] points. ${ }^{39}$ At $\bar{X}$ point, a surface state lies between 1.5 and $2.5 \mathrm{eV}$ above the Fermi level, ${ }^{39,40}$ but there is no associated occupied state to induce a direct transition (unless bulk states interfere). At $\bar{Y}$ point, a surface state just below the Fermi level ${ }^{41,42}$ may be coupled to an empty state above, whose location varies dramatically depending on the source from less than $1 \mathrm{eV}$ to around $2 \mathrm{eV}^{39-41}$ It also depends on the atomic surface structure, as the occupied state shifts up to above the Fermi level upon $(2 \times 1)$ reconstruction. ${ }^{41}$ Reflection anisotropy spectroscopy on the $(2 \times 1)$ reconstructed surface has evidenced a transition around $2.5 \mathrm{eV}$, polarized along $(\overline{1} 10) .{ }^{31,43} \mathrm{On} \mathrm{Au}(100)$, a state, probably related to d-bands, ${ }^{44}$ is described at $\overline{\mathrm{M}}$ point at $1.6 \mathrm{eV}$ below $\mathrm{E}_{\mathrm{F}}{ }^{45}$ on both normal and reconstructed surfaces, but no arrival state has been described for a surface transition. On the contrary, a Shockley state is located around $0.2 \mathrm{eV}$ below the Fermi level on the reconstructed surfaces at $\bar{X}$ point, ${ }^{46}$ while an empty state has been calculated around $2 \mathrm{eV}$ above $\mathrm{E}_{\mathrm{F}}{ }^{40}$ This leads to a surface transition with energies around $2.6 \mathrm{eV}$ in electroreflectance spectroscopy ${ }^{30}$ for the uncharged surface. In a general way, Ref. 40 has evidenced several surface transitions by electroreflectance for the three low index orientations of uncharged gold surfaces in the $2-3 \mathrm{eV}$ range and correlated them to calculated surface state transitions. Most states mentioned here have their counterpart on the more studied copper surfaces. ${ }^{47}$ Care must however be taken as selection rules apply for direct optical transitions between these states, which after careful inspection may appear polarization dependent or even forbidden. ${ }^{10,12,48}$

It is interesting to compare these results to silver. On $\operatorname{Ag}(111)$, the situation at $\bar{\Gamma}$ point is analogous to gold: a Shockley state just below the Fermi level, ${ }^{33,34}$ but no empty state above except an image state around $4 \mathrm{eV}^{49}$ as on $\mathrm{Cu}(111){ }^{12}$ At $\bar{M}$ point, the Tamm state is measured at $3.9 \mathrm{eV}$ below $\mathrm{E}_{\mathrm{F}}{ }^{50}$ Above the Fermi level at $\bar{M}$, the inverted bandgap of gold ${ }^{36}$ does not exist, ${ }^{49}$ excluding the existence of an empty surface state. On $\operatorname{Ag}(110)$, the situation at $\bar{Y}$ point is symmetric to gold, ${ }^{39}$ with a Shockley state just below the Fermi level ${ }^{42}$ and an empty state calculated around $1.6 \mathrm{eV}$ above $\mathrm{it}^{51}$ and measured around 1.7-1.8 eV..$^{4,52,53}$ This transition is responsible for the clear surface state transition resonance in SHG 
experiments. ${ }^{10}$ Finally, on $\operatorname{Ag}(100)$, a Shockley state is evidenced just below $(0.4 \mathrm{eV})$ the Fermi level at $\bar{X}$ point,, 54,55 but, contrary to gold, the upper Tamm state lies further away between 3.1 and $3.8 \mathrm{eV}^{49,55,56}$ As a consequence, there is no clear surface transition specific to low index silver surfaces in the visible range (1-3 eV), except on (110) faces. ${ }^{30,48}$ This can be understood as the transitions on (111) and (100) faces involve at least one state with a d-character. As compared to gold, they shift for silver to higher energies in the same way as the interband transitions for both metals. As such, the difference in the optical excitations of interband and surface state transitions between silver and gold stems from the common origin of their band structures.

The literature validates the existence of surface transitions which may account for the observed high SFG signal from gold on the three low index faces in the blue-green region. If we consider the (111) orientation as typical for the structural and optical properties of the polycrystalline gold surface (and also for silver ${ }^{57}$ ), then the analysis above also explains why the SFG signals from silver remain lower than for polycrystalline gold, all the more since (111) is also the most used orientation for SFG experiments on silver single crystals. Under this hypothesis, the transition at $\bar{M}$ point is the best candidate to account for our SFG data. A polycrystalline surface may also be considered as the scene where all allowed transitions between surface and bulk states may occur, without (or with little) wavevector selection. ${ }^{58}$ In this view, all the above-mentioned transitions may contribute to the experimental SFG (and SHG) enhancements, with the consequence that each resonance contributes for a small part of the processes (or equivalently involves a low number of electrons), leading to a total resonant enhancement less important than on a single crystal. This could explain why the surface state resonances mix with the free electron background on our data, while Urbach et al. ${ }^{10}$ only monitor the resonant signal by SHG on the $\mathrm{Ag}(110)$ single crystal. However, the fact that SHG on polycrystalline Ag remains the same as on $\mathrm{Ag}(111)$ over a broad range of energies does not support this hypothesis. ${ }^{59}$ Finally, the intrinsic widths of surface states are rather small [estimated, for example, at $21 \mathrm{meV}$ FWHM $\left(170 \mathrm{~cm}^{-1}\right)$ for the $\mathrm{Au}(111)$ Shockley state at $\bar{\Gamma}$ point $\left.{ }^{34}\right]$. This seems to contradict the widths fitted in Table II (by at least one order of magnitude), taking into account the negligible experimental linewidth of the visible beam for our SFG setup (around $10 \mathrm{~cm}^{-1}$ ). However, the experimental width measured by SFG integrates all the vertical transitions over the wavevector space, which may lead to a rather large broadening as a consequence of the relative dispersions of the initial and final states. In addition, a surface resonance, as found at $\bar{M}$ point on $\mathrm{Au}(111)$, may decay towards bulk states and has a larger width. ${ }^{8}$ Comparing a polycrystal and a single crystal on that point should give interesting clues on the validity of the surface state hypothesis.

Most surface states are calculated and measured on clean surfaces in controlled conditions. Our SFG data deal with a surface prepared and studied in air after modification by the adsorption of the thiol layer in an aqueous solution. It has been shown that surface adsorption dramatically modifies the surface state bands, shifting either their energies or their dispersions, ${ }^{41}$ inducing a structure change (e.g., reconstruction) which modifies full parts of the band structure ${ }^{60,61}$ or even removing them (for example, with $\mathrm{H}_{2} \mathrm{O},{ }^{56} \mathrm{H}_{2}, \mathrm{O}_{2}{ }^{52}$ ). However, RAS measurements in an electrochemical environment on $\mathrm{Au}(110)$ have shown that the observed surface transitions may survive upon adsorption of pyridine ${ }^{62}$ or sulfurcontaining amino acids. ${ }^{63}$ Adsorption of a thiol molecule on gold involves mostly electrons of the $6 \mathrm{~s}$ band to create the $\mathrm{Au}-\mathrm{S}$ bond, ${ }^{64}$ and we may consider that it will modify mostly the Shockley surface states. As for the transition at $\bar{M}$ point on the $\mathrm{Au}(111)$ surface, the lower state has been shown to be a Tamm state, but we could not find any clear information on the nature of the upper state. The influence of adsorption on this specific transition is therefore still unclear and should be investigated, both theoretically and experimentally.

\section{CONCLUSION AND PERSPECTIVES}

We have shown that both the intrinsic properties of gold as a material (the interband scenario) and a superposition of those with surface-specific properties (the effective surface state scenario) may account for the experimental SFG data on gold. The possibility of a surface state created by adsorption of the thiol monolayer has been discarded, and the surface transition dipole moment is most probably parallel to the interface. The interband description requires to artificially boost the interband response in order to compensate for overestimated free electron polarizabilities, in particular, in the infrared. As for the surface state scenario, it relies on the complex band structure of gold, giving rise to surface states and resonances when projected onto a surface orientation. Several transitions have been evidenced which may account for the observed behavior and for the discrepancies between gold and silver. Incomplete information on these processes, in particular, for a polycrystalline thiolated gold surface, does not allow to fully assess the validity of this scenario. At this stage, there is therefore no way to choose between these hypotheses. However, we have established that the free and bound electron densities overlap at the interface as a function of depth, with a possible free electron spill out (but no bound electron spill out).

In order to completely understand the surface processes involved in the second order nonlinear response of metals, our work provides a rather clear work program (as has been proposed in the past by Jiang et al. ${ }^{65}$ ) which may discriminate between the two scenarios above and help clarify the role of surface transitions. We suggest to record absolute SFG intensities for a fixed infrared wavelength and a few tunable visible wavelengths and to favor intensity comparisons between several interfaces recorded in the same conditions in order to avoid introducing experimental artifacts. First, a direct comparison between (110), polycrystalline and (111) orientations of bare silver crystals should clearly allow us to monitor the role of the (110) surface transitions and estimate the effect of polycrystallinity. Polarization and the azimuthal rotation angle should be tuned in order to control the characteristics of the transition dipole moment. Second, an absolute SFG intensity comparison between $\mathrm{Ag}(110)$ and several Au single crystal orientations would show whether the high gold signal has the same origin 
as for $\mathrm{Ag}(110)$. Third, the absolute comparison between bare and thiolated surfaces on the same crystal would also quantify the changes induced by surface adsorption. For the three experiments, the interband scenario should be responsible for a regular behavior (as a function of the visible wavelength) for a given metal, whereas the excitation of surface transitions will induce intense resonant signals on single crystals, with a strong dependence on the parameters (surface orientation, polarizations, angles, and surface coverage). Finally, ultrafast SFG setups could also provide additional information on the lifetimes of the processes involved in the resonant surface transitions (electron-hole pairs created by the interband transitions close to the surface, ${ }^{66,67}$ surface states ${ }^{34}$ ) and their link to the SFG signal enhancements in the visible range.

\section{SUPPLEMENTARY MATERIAL}

See supplementary material for the fitting of experimental data with one surface state and a constant nonresonant background, without Fresnel correction.

\section{APPENDIX A: EFFECTIVE NONLINEAR SUSCEPTIBILITY OF GOLD}

We recall here the results of Ref. 2

$$
\begin{gathered}
\chi_{\text {gold }}^{\text {eff }}=\chi_{\text {gold }}^{\text {eff,f }}+\chi_{\text {gold }}^{\text {eff, },} \\
\chi_{\text {gold }}^{\text {eff, } / \mathrm{b}}=F_{\text {zzz }} \chi_{S, z z z}^{\mathrm{f} / \mathrm{b}}-\mathrm{F}_{\mathrm{xxz}} \chi_{\mathrm{S}, \mathrm{xxz}}^{\mathrm{f} / \mathrm{b}}-\mathrm{F}_{\mathrm{xzx}} \chi_{\mathrm{S}, \mathrm{xzx}}^{\mathrm{f} / \mathrm{b}}+\mathrm{F}_{\mathrm{bulk}} \chi_{\mathrm{bulk}}^{\mathrm{f} / \mathrm{b}}, \\
\chi_{\mathrm{S}, \mathrm{zzz}}^{\mathrm{f}}=\frac{1}{2 \mathrm{n}_{0}^{\mathrm{f}} \mathrm{e}} \frac{\varepsilon_{1}^{\mathrm{f}}-1}{4 \pi \varepsilon_{1}^{\mathrm{f}}} \frac{1}{4 \pi \varepsilon_{2}^{\mathrm{f}}} \varepsilon_{1} \varepsilon_{2} \varepsilon_{3}\left[\mathrm{a}^{\mathrm{f}}\left(\omega_{1}, \omega_{2}\right)+\mathrm{a}_{\mathrm{s} 1}^{\mathrm{bf}}\left(\omega_{1}, \omega_{2}\right)\right], \\
\chi_{\mathrm{S}, \mathrm{zzz}}^{\mathrm{b}}=\frac{1}{2 \mathrm{n}_{0}^{\mathrm{b}} \mathrm{e}} \frac{\varepsilon_{1}^{\mathrm{b}}-1}{4 \pi \varepsilon_{1}^{\mathrm{b}}} \frac{1}{4 \pi \varepsilon_{2}^{\mathrm{b}}} \varepsilon_{1} \varepsilon_{2} \varepsilon_{3}\left[\mathrm{a}^{\mathrm{b}}\left(\omega_{1}, \omega_{2}\right)+\mathrm{a}_{\mathrm{s} 3}^{\mathrm{bf}}\left(\omega_{1}, \omega_{2}\right)\right],
\end{gathered}
$$

with terms $a^{b f}{ }_{s 1}$ (respectively, $a^{b f}{ }_{s 3}$ ) vanishing except for scheme 1 (respectively, scheme 3 )

$$
\begin{aligned}
\chi_{\mathrm{S}, \mathrm{xxz}}^{\mathrm{f} / \mathrm{b}} & =\frac{1}{2 \mathrm{n}_{0}^{\mathrm{f} / \mathrm{b}} \mathrm{e}} \frac{\varepsilon_{1}^{\mathrm{f} / \mathrm{b}}-1}{4 \pi} \frac{\varepsilon_{2}^{\mathrm{f} / \mathrm{b}}-1}{4 \pi} \frac{2 \omega_{1}}{\omega_{3}} \mathrm{~b}^{\mathrm{f} / \mathrm{b}}\left(\omega_{1}, \omega_{2}\right), \\
\chi_{\mathrm{S}, \mathrm{fzx}}^{\mathrm{f} / \mathrm{b}} & =\frac{1}{2 \mathrm{n}_{0} \mathrm{f} / \mathrm{b} e} \frac{\varepsilon_{1}^{\mathrm{f} / \mathrm{b}}-1}{4 \pi} \frac{\varepsilon_{2}^{\mathrm{f} / \mathrm{b}}-1}{4 \pi} \frac{2 \omega_{2}}{\omega_{3}} \mathrm{~b}^{\mathrm{f} / \mathrm{b}}\left(\omega_{2}, \omega_{1}\right) .
\end{aligned}
$$

Parameters $b^{\mathrm{f} / \mathrm{b}}$ equal -1 , whereas the values for parameters $a^{\mathrm{f} / \mathrm{b}}$, the Fresnel factors $F_{i j k}$, and the bulk terms for free and bound electrons, may be found in Paper II of the series, ${ }^{2}$ and the associated effective nonlinear susceptibilities in the literature. $4,68,69$ They all bear a prefactor $\frac{1}{2 \mathrm{n}_{0}^{\mathrm{f} / \mathrm{b} e}}$.
The total effective nonlinear susceptibility of gold may therefore be written as $\chi_{\mathrm{gold}}^{\text {eff }}=\frac{1}{2 \mathrm{n}_{0}^{\mathrm{f}} \mathrm{e}}$ [free electron terms $+\mathrm{R}_{\mathrm{bf}}$ bound electron terms $]$ with

$$
\mathrm{R}_{\mathrm{bf}}=\frac{\mathrm{n}_{0}^{\mathrm{f}}}{\mathrm{n}_{0}^{\mathrm{b}}}
$$

\section{APPENDIX B: FORM OF THE FIT FUNCTION FOR THE SURFACE STATE}

The general formulation for the nonlinear second-order susceptibility may be found in the textbooks. ${ }^{70}$ For a system initially in the ground state $\mathrm{g}$, we may write (with $\omega_{1}=\omega_{\text {vis }}$, $\omega_{2}=\omega_{\mathrm{IR}}$ and $\left.\omega_{3}=\omega_{1}+\omega_{2}=\omega_{\mathrm{SFG}}\right)$

$$
\begin{aligned}
& \chi_{\mathrm{ijk}}^{(2)}\left(\omega_{3} ; \omega_{1}, \omega_{2}\right) \\
& =\frac{\mathrm{N}}{2 \hbar} \sum_{\mathrm{m}, \mathrm{n}} \frac{\mu_{\mathrm{g} \leftarrow \mathrm{n}}^{\mathrm{i}} \mu_{\mathrm{n} \leftarrow \mathrm{m}}^{\mathrm{k}} \mu_{\mathrm{m} \leftarrow \mathrm{g}}^{\mathrm{j}}}{\left(\omega_{3}-\omega_{\mathrm{ng}}+\mathrm{i} \Gamma_{\mathrm{ng}}\right)\left(\omega_{1}-\omega_{\mathrm{mg}}+\mathrm{i} \Gamma_{\mathrm{mg}}\right)} \\
& +\frac{\mu_{\mathrm{g} \leftarrow \mathrm{n}}^{\mathrm{i}} \mu_{\mathrm{n} \leftarrow \mathrm{m}}^{\mathrm{j}} \mu_{\mathrm{m} \leftarrow \mathrm{g}}^{\mathrm{k}}}{\left(\omega_{3}-\omega_{\mathrm{ng}}+\mathrm{i} \Gamma_{\mathrm{ng}}\right)\left(\omega_{2}-\omega_{\mathrm{mg}}+\mathrm{i} \Gamma_{\mathrm{mg}}\right)} \\
& +\frac{\mu_{\mathrm{g} \leftarrow \mathrm{n}}^{\mathrm{j}} \mu_{\mathrm{n} \leftarrow \mathrm{m}}^{\mathrm{k}} \mu_{\mathrm{m} \leftarrow \mathrm{g}}^{\mathrm{i}}}{\left(\omega_{3}+\omega_{\mathrm{mg}}+\mathrm{i} \Gamma_{\mathrm{mg}}\right)\left(\omega_{1}+\omega_{\mathrm{ng}}+\mathrm{i} \Gamma_{\mathrm{ng}}\right)} \\
& +\frac{\mu_{\mathrm{g} \leftarrow \mathrm{n}}^{\mathrm{k}} \mu_{\mathrm{n} \leftarrow \mathrm{m}}^{\mathrm{j}} \mu_{\mathrm{m} \leftarrow \mathrm{g}}^{\mathrm{i}}}{\left(\omega_{3}+\omega_{\mathrm{mg}}+\mathrm{i} \Gamma_{\mathrm{mg}}\right)\left(\omega_{2}+\omega_{\mathrm{ng}}+\mathrm{i} \Gamma_{\mathrm{ng}}\right)} \\
& -\frac{\mu_{\mathrm{g} \leftarrow \mathrm{n}}^{\mathrm{k}} \mu_{\mathrm{n} \leftarrow \mathrm{m}}^{\mathrm{i}} \mu_{\mathrm{m} \leftarrow \mathrm{g}}^{\mathrm{j}}}{\left(\omega_{3}+\omega_{\mathrm{nm}}+\mathrm{i} \Gamma_{\mathrm{nm}}\right)\left(\omega_{1}-\omega_{\mathrm{mg}}+\mathrm{i} \Gamma_{\mathrm{mg}}\right)} \\
& -\frac{\mu_{\mathrm{g} \leftarrow \mathrm{n}}^{\mathrm{j}} \mu_{\mathrm{n} \leftarrow \mathrm{m}}^{\mathrm{i}} \mu_{\mathrm{m} \leftarrow \mathrm{g}}^{\mathrm{k}}}{\left(\omega_{3}+\omega_{\mathrm{nm}}+\mathrm{i} \Gamma_{\mathrm{nm}}\right)\left(\omega_{2}-\omega_{\mathrm{mg}}+\mathrm{i} \Gamma_{\mathrm{mg}}\right)} \\
& -\frac{\mu_{\mathrm{g} \leftarrow \mathrm{n}}^{\mathrm{j}} \mu_{\mathrm{n} \leftarrow \mathrm{m}}^{\mathrm{i}} \mu_{\mathrm{m} \leftarrow \mathrm{g}}^{\mathrm{k}}}{\left(\omega_{3}-\omega_{\mathrm{mn}}+\mathrm{i} \Gamma_{\mathrm{mn}}\right)\left(\omega_{1}+\omega_{\mathrm{ng}}+\mathrm{i} \Gamma_{\mathrm{ng}}\right)} \\
& -\frac{\mu_{\mathrm{g} \leftarrow \mathrm{n}}^{\mathrm{k}} \mu_{\mathrm{n} \leftarrow \mathrm{m}}^{\mathrm{i}} \mu_{\mathrm{m} \leftarrow \mathrm{g}}^{\mathrm{j}}}{\left(\omega_{3}-\omega_{\mathrm{mn}}+\mathrm{i} \Gamma_{\mathrm{mn}}\right)\left(\omega_{2}+\omega_{\mathrm{ng}}+\mathrm{i} \Gamma_{\mathrm{ng}}\right)} .
\end{aligned}
$$

Considering that the IR photons at the energies involved in the experiment may not excite any resonant transition in gold, the terms which may become resonant with the excitation of an electronic transition correspond to lines 1 and 2 for the SFG beam and 1 and 5 for the visible beam, while term 1 may become doubly resonant.

For a doubly resonant process to occur, it should involve the surface state $(\mathrm{m} \equiv \mathrm{SS})$ and a second state of higher energy $(\mathrm{n} \equiv \mathrm{e})$. Under the hypothesis of a single surface state at the gold surface, we have $\mathrm{n}=\mathrm{e}=\mathrm{SS}$, which means that the IR photon induces the $\mathrm{m} \rightarrow \mathrm{n}$ transition inside the same surface state. Considering that this state has no vibrational structure, this IR transition must remain purely of electronic nature. The surface state having a dispersion relationship in the $(\mathbf{k}, \mathrm{E})$ space, the IR photon must change the electronic momentum $\mathbf{k}$ in order to induce a jump in energy. Under the usual approximation 
of a photon with negligible momentum, this direct transition is impossible. A mediated transition, through a phonon, a defect, or an impurity, is in principle conceivable. For a fcc monoatomic crystal, there are no optical phonons, ${ }^{71}$ and considering a low-defect interface, the mediated transition cross section shall be negligible. As a consequence, the IR photon may not allow the doubly resonant process to occur and such a process will be neglected.

For a process singly resonant with $\omega_{3}$, we have

$$
\begin{aligned}
\chi_{\mathrm{ijk}}^{(2)}\left(\omega_{3} ; \omega_{1}, \omega_{2}\right)= & \frac{\mathrm{N}}{2 \hbar} \frac{\mu_{\mathrm{g} \leftarrow \mathrm{SS}}^{\mathrm{i}}}{\omega_{3}-\omega_{S S g}+\mathrm{i} \Gamma_{\mathrm{SSg}}} \sum_{\mathrm{m}} \frac{\mu_{\mathrm{SS} \leftarrow \mathrm{m}}^{\mathrm{k}} \mu_{\mathrm{m} \leftarrow \mathrm{g}}^{\mathrm{j}}}{\omega_{1}-\omega_{\mathrm{mg}}+\mathrm{i} \Gamma_{\mathrm{mg}}} \\
& +\frac{\mu_{\mathrm{SS} \leftarrow \mathrm{m}}^{\mathrm{j}} \mu_{\mathrm{m} \leftarrow \mathrm{g}}^{\mathrm{k}}}{\omega_{2}-\omega_{\mathrm{mg}}+\mathrm{i} \Gamma_{\mathrm{mg}}},
\end{aligned}
$$

and for a resonance at $\omega_{1}$

$$
\begin{aligned}
\chi_{\mathrm{jik}}^{(2)}\left(\omega_{3} ; \omega_{1}, \omega_{2}\right)= & \frac{\mathrm{N}}{2 \hbar} \frac{\mu_{\mathrm{SS} \leftarrow \mathrm{g}}^{\mathrm{i}}}{\omega_{1}-\omega_{\mathrm{SSg}}+\mathrm{i} \Gamma_{\mathrm{SSg}}} \sum \frac{\mu_{\mathrm{g} \leftarrow \mathrm{m}}^{\mathrm{j}} \mu_{\mathrm{m} \leftarrow \mathrm{SS}}^{\mathrm{k}}}{\omega_{3}-\omega_{\mathrm{mg}}+\mathrm{i} \Gamma_{\mathrm{mg}}} \\
& -\frac{\mu_{\mathrm{g} \leftarrow \mathrm{m}}^{\mathrm{k}} \mu_{\mathrm{m} \leftarrow \mathrm{SS}}^{\mathrm{j}}}{\omega_{3}+\omega_{\mathrm{mSS}}+\mathrm{i} \Gamma_{\mathrm{mSS}}} .
\end{aligned}
$$

We now consider the possibility to excite the surface state with the SFG and the visible beams, simultaneously. In the (ppp) configuration, $(\mathrm{i}, \mathrm{j}, \mathrm{k})$ stand either for $\mathrm{x}$ or $\mathrm{z}$, and for an in-plane isotropic symmetry as considered here, the only nonvanishing coefficients are xxz, xzx, Zxx, and zzz. In addition, exciting the surface state requires that the electric field is aligned along the transition dipole moment, i.e., either in-plane (x) or normal to the surface $(\mathrm{z})$. The $\chi^{(2)}$ components resonant with the SS transition will therefore differ whether it is excited by the SFG or the visible photon, and the simultaneous excitation requires to compare the (ijk) coefficient in (B2) with the (jik) coefficient in (B3).

At this stage, we may make a rather rough approximation in order to compare the amplitudes of both terms. We limit the summation over $m$ to high energy states only (called $\alpha$ ), considering that there are indeed resonant states in the UV for the system, i.e., far from and higher than the photon energies involved in the SFG process. We thus neglect $\omega_{1}, \omega_{2}, \omega_{3}$, and the widths in front of their energies $\omega_{\alpha}$. The $\omega_{3}$-resonant term becomes

$$
\begin{aligned}
\chi_{\mathrm{ijk}}^{(2)}\left(\omega_{3} ; \omega_{1}, \omega_{2}\right) \approx & -\frac{\mathrm{N}}{2 \hbar} \frac{\mu_{\mathrm{g} \leftarrow \mathrm{SS}}^{\mathrm{i}}}{\omega_{3}-\omega_{\mathrm{SSg}}+\mathrm{i} \Gamma_{\mathrm{SSg}}} \\
& \times \sum_{\alpha} \frac{1}{\omega_{\alpha}}\left(\mu_{\mathrm{SS} \leftarrow \alpha}^{\mathrm{k}} \mu_{\alpha \leftarrow \mathrm{g}}^{\mathrm{j}}+\mu_{\mathrm{SS} \alpha \alpha}^{\mathrm{j}} \mu_{\alpha \leftarrow \mathrm{g}}^{\mathrm{k}}\right),
\end{aligned}
$$

and the $\omega_{1}$-resonant term becomes

$$
\begin{aligned}
\chi_{\mathrm{jik}}^{(2)}\left(\omega_{3} ; \omega_{1}, \omega_{2}\right) \approx & -\frac{\mathrm{N}}{2 \hbar} \frac{\mu_{\mathrm{SS} \leftarrow \mathrm{g}}^{\mathrm{i}}}{\omega_{1}-\omega_{\mathrm{SSg}}+\mathrm{i} \Gamma_{\mathrm{SSg}}} \\
& \times \sum_{\alpha} \frac{1}{\omega_{\alpha}}\left(\mu_{\alpha \leftarrow \mathrm{SS}}^{\mathrm{k}} \mu_{\mathrm{g} \leftarrow \alpha}^{\mathrm{j}}+\mu_{\alpha \leftarrow \mathrm{SS}}^{\mathrm{j}} \mu_{\mathrm{g} \leftarrow \alpha}^{\mathrm{k}}\right) .
\end{aligned}
$$

Putting both terms together, we see that the numerators are conjugate complex numbers, thus the fit function for the surface state must have the following form:

$$
\chi=\frac{\mathrm{Be}^{\mathrm{i} \varphi_{\mathrm{SFG}}}}{\omega_{\mathrm{SFG}}-\omega_{\mathrm{SS}}+\mathrm{i} \Gamma_{\mathrm{SS}}}+\frac{\mathrm{Ce}^{\mathrm{i} \varphi_{\mathrm{vis}}}}{\omega_{\mathrm{vis}}-\omega_{\mathrm{SS}}+\mathrm{i} \Gamma_{\mathrm{SS}}},
$$

with $\mathrm{B}=\mathrm{C}$ and $\varphi_{\text {vis }}=-\varphi_{\text {SFG }}$.

As explained in the text, the approximation of high energies used here is not fully enforced, so amplitudes and phases obtained from curve fitting may deviate from this ideal picture.

${ }^{1}$ L. Dalstein, A. Revel, C. Humbert, and B. Busson, J. Chem. Phys. 148, 134701 (2018).

${ }^{2}$ B. Busson and L. Dalstein, J. Chem. Phys. 149, 034701 (2018).

${ }^{3}$ A. Tadjeddine and A. Le Rille, Electrochim. Acta 45, 601 (1999).

${ }^{4}$ J. A. Maytorena, B. S. Mendoza, and W. L. Mochán, Phys. Rev. B 57, 2569 (1998).

${ }^{5}$ B. S. Mendoza, W. L. Mochán, and J. A. Maytorena, Phys. Rev. B 60, 14334 (1999).

${ }^{6}$ T. A. Ishibashi and H. Onishi, Appl. Phys. Lett. 81, 1338 (2002).

${ }^{7}$ S. G. Davison and M. Steslicka, Basic Theory of Surface States (Oxford University Press, New York, 1992).

${ }^{8}$ S. D. Kevan, in Electronic Structure, edited by K. Horn and M. Scheffler (Elsevier, Amsterdam, NL, 2000), pp. 433-510.

${ }^{9}$ Y. R. Shen, Annu. Rev. Phys. Chem. 40, 327 (1989).

${ }^{10}$ L. E. Urbach, K. L. Percival, J. M. Hicks, E. W. Plummer, and H. L. Dai, Phys. Rev. B 45, 3769 (1992).

${ }^{11}$ H. Tanaka, H. Wakimoto, T. Miyazaki, G. Mizutani, and S. Ushioda, Surf. Sci. 427-428, 147 (1999).

${ }^{12}$ G. Lupke, D. J. Bottomley, and H. M. van Driel, Phys. Rev. B 49, 17303 (1994).

${ }^{13}$ K. C. Chou, S. Westerberg, Y. R. Shen, P. N. Ross, and G. A. Somorjai, Phys. Rev. B 69, 153413 (2004).

${ }^{14}$ M. Guerrisi, R. Rosei, and P. Winsemius, Phys. Rev. B 12, 557 (1975).

${ }^{15}$ R. L. Olmon, B. Slovick, T. W. Johnson, D. Shelton, S. H. Oh, G. D. Boreman, and M. B. Raschke, Phys. Rev. B 86, 235147 (2012).

${ }^{16}$ N. E. Christensen and B. O. Seraphin, Phys. Rev. B: Solid State 4, 3321 (1971).

${ }^{17}$ J. A. Maytorena, W. L. Mochán, and B. S. Mendoza, Phys. Rev. B 57, 2580 (1998).

${ }^{18}$ P. Guyot-Sionnest, A. Tadjeddine, and A. Liebsch, Phys. Rev. Lett. 64, 1678 (1990).

${ }^{19}$ B. Busson and A. Tadjeddine, J. Phys. Chem. C 113, 21895 (2009).

${ }^{20}$ P. A. Covert and D. K. Hore, J. Phys. Chem. C 119, 271 (2015).

${ }^{21}$ E. Sani and A. Dell'Oro, Opt. Mater. 60, 137 (2016).

${ }^{22}$ S. M. McGinty, M. K. Kapala, and R. F. Niedziela, Phys. Chem. Chem. Phys. 11, 7998 (2009).

${ }^{23}$ B. H. Yan, B. Stadtmuller, N. Haag, S. Jakobs, J. Seidel, D. Jungkenn, S. Mathias, M. Cinchetti, M. Aeschlimann, and C. Felser, Nat. Commun. 6, 10167 (2015).

${ }^{24}$ P. M. Sheverdyaeva, R. Requist, P. Moras, S. K. Mahatha, M. Papagno, L. Ferrari, E. Tosatti, and C. Carbone, Phys. Rev. B 93, 035113 (2016).

${ }^{25}$ J. R. Smith, F. J. Arlinghaus, and J. G. Gay, Phys. Rev. B 22, 4757 (1980).

${ }^{26}$ A. Euceda, D. M. Bylander, L. Kleinman, and K. Mednick, Phys. Rev. B 27, 659 (1983).

${ }^{27}$ E. Wimmer and A. J. Freeman, in Electronic Structure, edited by K. Horn and M. Scheffler (Elsevier, Amsterdam, NL, 2000), pp. 1-91.

${ }^{28}$ H. Lüth, Solid Surfaces, Interfaces and Thin Films (Springer Verlag, Berlin, Germany, 2001).

${ }^{29}$ S. Hufner, Photoelectron Spectroscopy Principles and Applications (Springer Verlag, Berlin, Germany, 2003).

${ }^{30}$ W. Boeck and D. M. Kolb, Surf. Sci. 118, 613 (1982).

${ }^{31}$ P. Weightman, D. S. Martin, R. J. Cole, and T. Farrell, Rep. Prog. Phys. 68, 1251 (2005).

${ }^{32}$ B. Bozzini, B. Busson, G. Pietro De Gaudenzi, C. Mele, and A. Tadjeddine, J. Solid State Electrochem. 12, 303 (2008).

${ }^{33}$ S. D. Kevan and R. H. Gaylord, Phys. Rev. B 36, 5809 (1987).

${ }^{34}$ F. Reinert, G. Nicolay, S. Schmidt, D. Ehm, and S. Hufner, Phys. Rev. B 63, 115415 (2001).

${ }^{35}$ D. P. Woodruff, W. A. Royer, and N. V Smith, Phys. Rev. B 34, 764 (1986).

${ }^{36}$ N. Takeuchi, C. T. Chan, and K. M. Ho, Phys. Rev. B 43, 13899 (1991). 
${ }^{37}$ B. Metzger, L. L. Gui, and H. Giessen, Opt. Lett. 39, 5293 (2014).

${ }^{38}$ R. Mazzarello, A. Dal Corso, and E. Tosatti, Surf. Sci. 602, 893 (2008).

${ }^{39}$ R. A. Bartynski and T. Gustafsson, Phys. Rev. B 33, 6588 (1986).

${ }^{40}$ S. H. Liu, C. Hinnen, C. N. V Huong, N. R. Detacconi, and K. M. Ho, J. Electroanal. Chem. 176, 325 (1984).

${ }^{41}$ A. Nuber, M. Higashiguchi, F. Forster, P. Blaha, K. Shimada, and F. Reinert, Phys. Rev. B 78, 195412 (2008).

${ }^{42}$ R. Courths, H. Wern, U. Hau, B. Cord, V. Bachelier, and S. Hufner, J. Phys. F: Met. Phys. 14, 1559 (1984).

${ }^{43}$ D. S. Martin, N. P. Blanchard, and P. Weightman, Surf. Sci. 532, 1 (2003).

${ }^{44}$ P. Heimann, J. Hermanson, H. Miosga, and H. Neddermeyer, Phys. Rev. B 20, 3059 (1979).

${ }^{45}$ P. Heimann, J. Hermanson, H. Miosga, and H. Neddermeyer, Phys. Rev. Lett. 43, 1757 (1979).

${ }^{46}$ S. Bengio, V. Navarro, M. A. Gonzalez-Barrio, R. Cortes, I. Vobornik, E. G. Michel, and A. Mascaraque, Phys. Rev. B 86, 045426 (2012).

${ }^{47}$ W. Jacob, V. Dose, U. Kolac, T. Fauster, and A. Goldmann, Z. Phys. B: Condens. Matter 63, 459 (1986).

${ }^{48}$ K. M. Ho, C. L. Fu, S. H. Liu, D. M. Kolb, and G. Piazza, J. Electroanal. Chem. 150, 235 (1983).

${ }^{49}$ A. Goldmann, V. Dose, and G. Borstel, Phys. Rev. B 32, 1971 (1985).

${ }^{50}$ R. Courths, H. Wern, and S. Hufner, Solid State Commun. 61, 257 (1987).

${ }^{51}$ K. M. Ho, B. N. Harmon, and S. H. Liu, Phys. Rev. Lett. 44, 1531 (1980).

${ }^{52}$ B. Reihl, R. R. Schlittler, and H. Neff, Phys. Rev. Lett. 52, 1826 (1984).

${ }^{53}$ B. Reihl, R. R. Schlittler, and H. Neff, Surf. Sci. 152, 231 (1985).

${ }^{54}$ L. Savio, L. Vattuone, M. Rocca, V. De Renzi, S. Gardonio, C. Mariani, U. del Pennino, G. Cipriani, A. Dal Corso, and S. Baroni, Surf. Sci. 486, 65 (2001).
${ }^{55}$ D. M. Kolb, W. Boeck, K. M. Ho, and S. H. Liu, Phys. Rev. Lett. 47, 1921 (1981).

${ }^{56}$ B. Reihl, K. H. Frank, and R. R. Schlittler, Phys. Rev. B 30, 7328 (1984).

${ }^{57}$ Y. Borensztein, Surf. Sci. 177, 353 (1986).

${ }^{58}$ B. Reihl and R. R. Schlittler, Phys. Rev. B 29, 2267 (1984).

${ }^{59}$ C. Matranga and P. Guyot-Sionnest, J. Chem. Phys. 115, 9503 (2001).

${ }^{60}$ L. H. Tjeng, M. B. J. Meinders, and G. A. Sawatzky, Surf. Sci. 233, 163 (1990).

${ }^{61}$ F. Henglein, D. M. Kolb, L. Stolberg, and J. Lipkowski, Surf. Sci. 291, 325 (1993).

${ }^{62}$ C. I. Smith, A. J. Maunder, C. A. Lucas, R. J. Nichols, and P. Weightman, J. Electrochem. Soc. 150, E233 (2003).

${ }^{63}$ R. LeParc, C. I. Smith, M. C. Cuquerella, R. L. Williams, D. G. Fernig, C. Edwards, D. S. Martin, and P. Weightman, Langmuir 22, 3413 (2006).

${ }^{64}$ M. Tachibana, K. Yoshizawa, A. Ogawa, H. Fujimoto, and R. Hoffmann, J. Phys. Chem. B 106, 12727 (2002).

${ }^{65}$ M. Y. Jiang, G. Pajer, and E. Burstein, Surf. Sci. 242, 306 (1991).

${ }^{66}$ S. Hagen, P. Kate, F. Leyssner, D. Nandi, M. Wolf, and P. Tegeder, J. Chem. Phys. 129, 164102 (2008)

${ }^{67}$ E. Knoesel, A. Hotzel, and M. Wolf, Phys. Rev. B 57, 12812 (1998).

${ }^{68}$ A. V Petukhov, Phys. Rev. B 52, 16901 (1995).

${ }^{69}$ N. Bloembergen and P. S. Pershan, Phys. Rev. 128, 606 (1962).

${ }^{70}$ R. W. Boyd, Nonlinear Optics (Academic Press, San Diego, CA, USA, 2003).

${ }^{71}$ C. Kittel, Introduction to Solid State Physics (John Wiley and Sons, New York, NY, USA, 1976). 